\title{
Quantenphysik in der Schule - Was benötigen Lehrkräfte? Ergebnisse einer Delphi-Studie
}

\author{
Kim-Alessandro Weber ${ }^{1}$ (D) - Gunnar Friege ${ }^{2} \cdot$ Rüdiger Scholz $^{1}$ \\ Eingegangen: 4. Juli 2019 / Angenommen: 20. September 2020 / Online publiziert: 13. Oktober 2020 \\ (C) Der/die Autor(en) 2020
}

\section{Zusammenfassung}

Das Themengebiet Quantenphysik gilt als gleichermaßen bedeutend wie herausfordernd für den Physikunterricht in der Schule. Die Erzeugung einer empirischen Basis für die Konstruktion von Leitlinien für eine im besonderen Maße adressatenspezifische Lehrerfortbildung zum Thema Quantenphysik ist Gegenstand dieses Beitrags.

Die Didaktische Rekonstruktion zur Erarbeitung von Leitlinien für Lehrerfortbildungen leitet die vorgestellte Untersuchung. Basis der Rekonstruktion bilden die Analyse von bestehenden Konzepten zum Quantenphysikunterricht in der Schule und die empirische Erhebung von Sichtweisen der Lehrenden auf diese. Der Fokus liegt in diesem Beitrag auf der Erhebung und Auswertung der Sichtweisen von Lehrenden. Zu diesem Zweck wurde eine iterative Kommunikationstechnik zur Erhebung und Quantifizierung von Expertenmeinungen verwendet, die Delphi-Methode, welche in der fachdidaktischen Forschung etabliert ist.

Als Ergebnis der Didaktischen Rekonstruktion werden sechs Leitlinien vorgestellt, die Dozenten bei der Planung und Durchführung der Fortbildung unterstützen. Die Leitlinien implizieren eine Fortbildung, die den Weg von der klassischen zur Quantenphysik beschreitet und dabei Schlüsselexperimente in das Zentrum stellt.

Schlüsselwörter Quantenphysik · Lehrerfortbildung · Didaktische Rekonstruktion · Delphi-Methode

\section{Quantum Physics at School—What do Teachers need? Results of a Delphi Study}

\begin{abstract}
Quantum physics is considered to be equally important and challenging for teaching physics at school. This paper aims to provide an empirical basis for the construction of guidelines for a teacher training course on quantum physics, which is particularly focused on teachers' needs.

The educational reconstruction for teacher education leads the presented investigation. The reconstruction is based on the analysis of existing concepts for quantum physics teaching in schools and the empirical collection of teachers' views on them. The focus of this paper is on the collection and evaluation of teachers' views. For this purpose, an iterative communication technique was used to collect and quantify expert opinions, the Delphi method, which is established in didactic research.

As a result of the educational reconstruction, six guidelines are presented which support the lecturer in planning and conducting the teacher training. The guidelines imply an advanced training program that follows the path from classical to quantum physics with special emphasis on key experiments.
\end{abstract}

Keywords Quantum Physics · Teacher Training · Educational Reconstruction · Delphi Method

Kim-Alessandro Weber

weber@iqo.uni-hannover.de

1 Institut für Quantenoptik, Leibniz Universität Hannover, Welfengarten 1, 30167 Hannover, Deutschland
2 Institut für Didaktik der Mathematik und Physik, AG Physikdidaktik, Leibniz Universität Hannover, Welfengarten 1A, 30167 Hannover, Deutschland 


\section{Quantenphysik: Unterrichtliche und gesellschaftliche Relevanz}

Quantenphysik eignet sich im besonderen Maß für die Einnahme einer Metaperspektive auf Naturwissenschaften. Ihre Vergangenheit gilt als exemplarisch für einen außergewöhnlichen Theoriewechsel in der Physik mit einem breiten Anwendungsbereich: Die Theorie verändert unsere Weltanschauung und darauf aufbauende Technologien werden unser alltägliches Leben zukünftig noch stärker verändern, als schon bereits in den vergangenen Jahrzehnten.

Die Kultusministerkonferenz (2004) sehen in den Einheitlichen Prüfungsanforderungen in der Abiturprüfung $(E P A)$ die Quantenphysik als ein verbindliches und grundlegendes Thema für die Abiturprüfung vor:

Grundlegende Merkmale von Quantenobjekten unter Einbezug erkenntnistheoretischer Aspekte Wellenmerkmal, Quantenmerkmal, stochastisches Verhalten, Komplementarität, Nichtlokalität, Verhalten beim Messprozess. (Kultusministerkonferenz 2004)

Die explizite Nennung der ,erkenntnistheoretischen Aspekte" unterstreicht die Einnahme der Metaperspektive auf Naturwissenschaften und den Erkenntnisprozess. Dies stellt eine herausfordernde Aufgabe für den Physikunterricht in der Schule dar: Beispielsweise stellt sich die Frage nach einem Schlüsselexperiment (Laumann et al. 2019) für die Schule, welches die Grenzen der klassischen Theorie aufdeckt. Zudem muss eine Positionierung des Unterrichts zur Falsifikation der klassischen Theorie erfolgen und hier im Speziellen die Behandlung des Dualismus-Begriffs im Unterricht geklärt werden. Eine Verwendung dieses Begriffs in der Art eines Schutzgürtels von Hilfshypothesen, die im Sinne von Lakatos (1974) um den harten Kern der alten Theorie gelegt werden, verschüttet den Erkenntnisprozess.

Die Reduktion auf ein als abstrakt und schwer empfundenes Themengebiet wird der Herausforderung bei einer Didaktisierung der Quantenphysik nicht gerecht. Das Potential der Quantenphysik für den Unterricht liegt darin, eine wissenschaftskritische Position zu entwickeln, Erkenntnisprozesse zu diskutieren und Physik als empirische Wissenschaft auszuzeichnen. Die Ausschöpfung des Potentials für den Physikunterricht ist in der Schulpraxis jedoch eine besondere Herausforderung und zwar insbesondere für Lehrende (Müller et al. 2000; Bronner 2010, S. 5; Pospiech und Schöne 2012). Gezielt für die Vermittlung von Quantenphysik im Unterricht, soll eine bedarfsorientierte Fortbildung zur Unterstützung von Lehrenden entwickelt werden. $\mathrm{Zu}$ diesem Zweck wird im hier beschriebenen Beitrag eine empirische Basis geschaffen: Die subjektive Sicht von Lehrenden auf die Quantenphysik im Schulunterricht. Hiervon ausgehend werden Leitlinien für eine Lehrerfortbildung abgeleitet.
Die Didaktische Rekonstruktion zur Erarbeitung von Leitlinien für eine Lehrerfortbildung nach Van Dijk und Kattmann (2007) sowie Nawrath (2010) ist dabei auch für die Erarbeitung von Leitlinien für eine Lehrerfortbildung und in der Folge für das Forschungsvorhaben strukturgebend. Die Studie wird mit der Delphi-Methode umgesetzt.

\section{Lehrerfortbildungen zur Quantenphysik in der fach didaktischen Forschung}

Der vorgestellte Beitrag stellt die Adressatenspezifität der Lehrerfortbildung in den Vordergrund. Dazu wird die subjektive Sicht der Lehrenden auf bestehende Konzepte zur Quantenphysik empirisch erhoben. Eine derart spezifische empirische Studie zur praktisch-unterrichtlichen Perspektive existiert derzeit nicht und stellt die Forschungslücke dar, die durch dieses Vorhaben geschlossen werden soll. Die so gewonnene empirische Basis ermöglicht anschließend die Konstruktion von Leitlinien einer Fortbildung und steuert die Auswahl, Restrukturierung und Kombination von bestehenden didaktischen Konzepten zur Quantenphysik als Inhalte der Fortbildung (z. B. Bronner 2010; Müller und Wiesner 2000; Küblbeck und Müller 2003; Zollman et al. 2002). Hierfür stellt die Didaktische Rekonstruktion zur Erarbeitung von Leitlinien für die Lehrerfortbildung die theoretische Rahmung bereit (vgl. Van Dijk und Kattmann 2007). Durch diese besondere Berücksichtigung der praktischen Perspektive soll eine hohe Unterrichtsrelevanz sichergestellt werden. Diese ist laut Darling-Hammond et al. (2009) ein Grundsatz für eine hohe Transferwirkung der Fortbildungsinhalte in den Unterricht der Teilnehmenden. Unterstrichen wird dieser Grundsatz ebenfalls von Wolf et al. (1999) sowie Lipowsky und Rzejak (2012).

\section{Didaktische Rekonstruktion zur Erarbeitung von Leitlinien für die Lehrerfortbildung}

Die Didaktische Rekonstruktion geht auf Kattmann et al. (1997) zurück und ist ein Modell zur didaktischen Konzeption eines Themas, das als Bezugsrahmen in der Physikdidaktik und anderen Didaktiken etabliert ist (vgl. Krüger et al. 2014). Hierbei wird die Beziehung von Fachlicher Klärung, dem Erfassen von Schülerperspektiven und der Didaktischen Strukturierung eines Themas beschrieben. Die Fachliche Klärung und das Erfassen von Schülerperspektiven bilden die Basis der Rekonstruktion. Die wechselseitige und gegenseitige Bezugnahme dieser beiden Basiskomponenten ermöglicht die didaktische Strukturierung eines Themas, die Schülerperspektiven und die Fachliche Klärung berücksichtigt. 
Abb. 1 Modell der Didaktischen Rekonstruktion zur Konzeption von Unterricht (unten links) als Teil des ERTE-Modells. (Nach Nawrath 2010; sowie Van Dijk und Kattmann 2007)

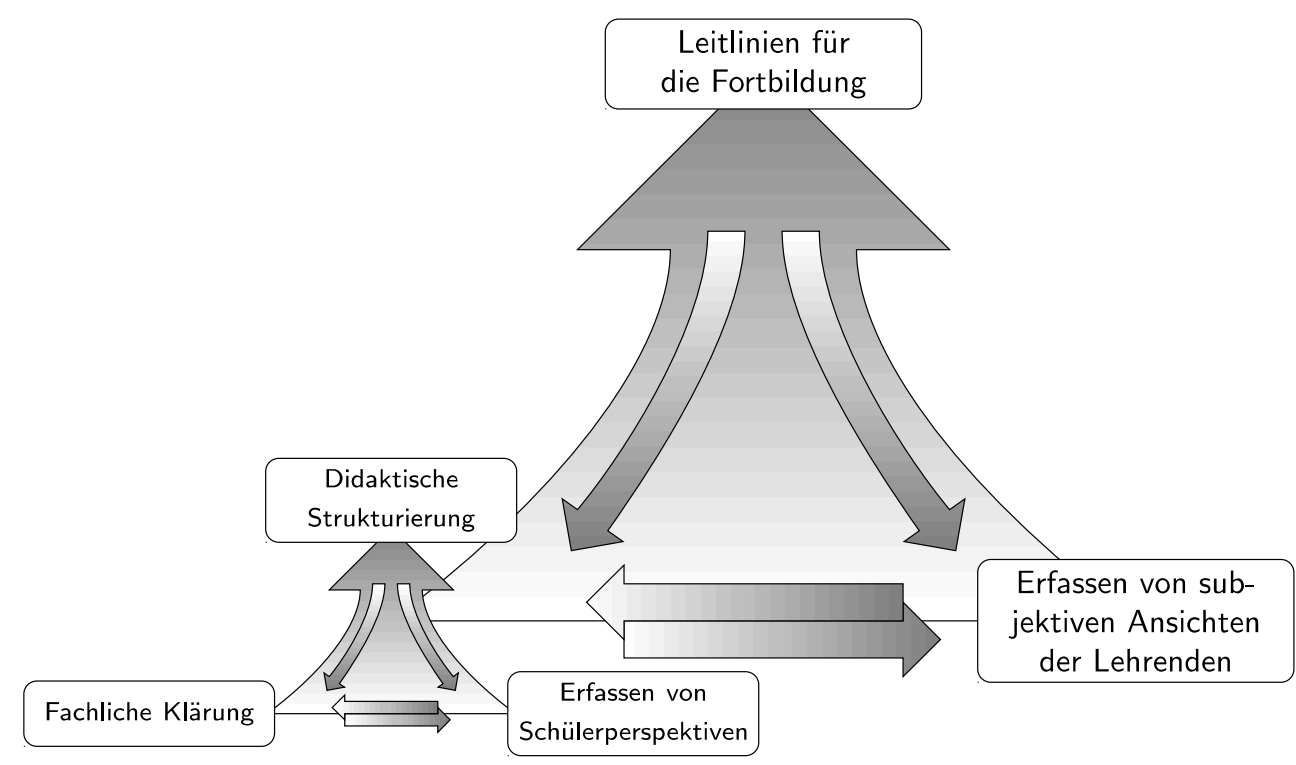

Van Dijk und Kattmann (2007) stellen mit dem ERTE-Modell (Educational Reconstruction for Teacher Education) eine Erweiterung des Modells der Didaktischen Rekonstruktion vor, welche zur Entwicklung von Fortbildungen für Lehrende genutzt werden kann. Nawrath (2010) konstruiert mit Hilfe des ERTE-Modells Leitlinien für eine Fortbildung zur Unterrichtsentwicklung unter Berücksichtigung von Kontexten. Die Basis des Didaktischen Triplets bildet hierbei die wechselseitige und gleichberechtigte Beziehung zwischen Didaktischen Konzeptionen und den Subjektiven Ansichten der Lehrenden zu diesen. Ausgehend von dieser Basis werden die Leitlinien für die Fortbildung konstruiert.

Die Modelle können ineinander verschachtelt werden (vgl. Abb. 1), wodurch das ERTE-Modell als Erweiterung der Didaktischen Rekonstruktion begriffen werden kann. Das Modell strukturiert das hier beschriebene Forschungsvorhaben, indem die subjektiven Ansichten und bestehenden fachdidaktischen Konzeptionen systematisch in den Fokus gestellt werden. Somit kann eine Fortbildung konzipiert werden, die im besonderen Maß den Bedarf von Lehrenden sowie deren Sicht auf didaktische Konzeptionen gerecht wird.

Ein Design-Based-Research-Ansatz (z.B. Reinmann 2005) wurde als Forschungsrahmen ebenfalls in Betracht gezogen: Zur Entwicklung einer initialen Fortbildung hätte jedoch ein elaborierter Hypothesenraum über den themenspezifischen Bedarf der Lehrenden bestehen müssen. Im ERTE-Modell war es schließlich naheliegender, ein hypothesengenerierendes und -testendes Verfahren (DelphiStudie) zu implementieren, um Leitlinien für die Fortbildung zu generieren.

\section{Ziele und Forschungsfragen}

Im ERTE-Modell muss zunächst die Basis des Triplets geschaffen werden. Hierbei muss einerseits eine Klärung der fachdidaktischen Konzepte als Resultat einer didaktischen Konzeption und andererseits die Erhebung der subjektiven Sicht der Lehrenden erfolgen:

Die Klärung der fachdidaktischen Konzepte kann als analytischer Prozess begriffen werden. Die Sichtung bestehender Konzepte soll an dieser Stelle ausgeführt werden. Drei Fragen leiten den Prozess der Klärung fachdidaktischer Konzepte zur Quantenphysik:

K1 Auf welchen Schwerpunkten und Prinzipien beruhen fachdidaktische Konzepte zum Schulunterricht in Quantenphysik?

K2 Welche empirischen Evidenzen gibt es für die Wirkung fachdidaktischer Konzepte in der Quantenphysik?

K3 Welchen Einfluss haben Lehrpläne, Standards und Zentralprüfungen auf die Nutzung der fachdidaktischen Konzepte?

Die Erfassung der subjektiven Ansichten von Lehrenden zu Konzepten in der Quantenphysik erfordert ein empirisches Vorgehen. Dieser Prozess wird hier ebenfalls durch drei Fragen geleitet:

S1 Welche fachdidaktischen Konzepte werden von Lehrkräften bei der Planung und Durchführung von Unterricht in der Quantenphysik berücksichtigt? 
S2 Welche Themen der Quantenphysik werden in der Unterrichtspraxis von den Lehrenden als besondere Herausforderung für die Lernenden wahrgenommen?

S3 Wie ist die retrospektive Sicht auf die eigene Ausbildung im Themengebiet Quantenphysik?

Die Konstruktion der Leitlinien erfolgt, indem die Konzepte und die subjektive Sicht der Lehrenden aufeinander bezogen werden. Dieser Prozess wird durch eine übergeordnete Forschungsfrage geleitet:

Wie sollte eine Fortbildung gestaltet sein, die

1. den Bedarf von Lehrkräften berücksichtigt und

2. die Lehrenden dazu befähigt, technologie- und forschungsrelevante Quantenphysik zu vermitteln sowie

3. deren erkenntnistheoretischen Ansprüchen gerecht wird?

Im Folgenden wird der Fokus auf die Erfassung subjektiver Ansichten zu den Konzepten sowie die Konstruktion der Leitlinien gelegt.

\section{Klärung fachdidaktischer Konzepte}

Die Klärung der fachdidaktischen Konzepte kann hier nur übersichtshalber gegeben werden und ist auf die Schwerpunktsetzung konzentriert. Hier wird demnach vor allem Frage K1 nachgegangen. Für eine ausführlichere Darstellung der Konzepte ist auf Weber (2018), Willer (2003) und für die Perspektive der Unterrichtspraxis auf Rode und Barth (2017) verwiesen.

Die Ebenen, auf die sich die Konzepte beziehen, sind sehr unterschiedlich. Einige Konzepte behandeln einzelne Unterrichtsangebote zu spezifischen Themen wie die Quantenkryptographie. Andere Konzepte beschreiben einen umfassenderen Zugang zur Quantenphysik, der auch Themen aufgreift, die über die in den Curricula der einzelnen Länder genannten Themen weit hinaus gehen. Zu den unterschiedlichen Konzepten existieren dabei kaum Evidenzen oder gar vergleichende Feldstudien (Willer 2003), sodass sich eine Bewertung und Auswahl der Konzepte für die Lehrkräfte in den Schulen schwierig gestalten und möglicherweise nur vom persönlichen Interesse bestimmt werden.

Um dieses facettenreiche Konzeptkonglomerat einzuordnen, schlagen Müller und Wiesner (2000) eine Kategorisierung nach Schwerpunktsetzungen (SPS) vor:

SPS a „Unterrichtskonzepte, die sich besonders auf die Prinzipien des quantenmechanischen Formalismus konzentrieren (z.B. Feynman-Zeiger) und diese auf verschiedene Fragestellungen anwenden." (Müller und Wiesner 2000, S. 1) (z. B. Feynman 2006; Bader 2000; Rode 2011; Werner, 2000)
SPS b „Unterrichtskonzepte, deren Schwerpunkt auf den begrifflichen Fragestellungen der Quantenphysik liegt.“ (Müller und Wiesner 2000, S. 1) (z. B. Müller und Wiesner 2000; Küblbeck und Müller 2003; Fischler 1992)

SPS c „Unterrichtskonzepte, in denen die Quantenphysik als Basis für das Verständnis zahlreicher physikalischer Theorien (z.B. Atomphysik, Kernphysik, Teilchenphysik, Festkörperphysik) begriffen wird.“ (Müller und Wiesner 2000, S. 1) (z. B. Niedderer 1992)

SPS d ,Unterrichtskonzepte, in denen sie als Grundlage für zahlreiche technologische Anwendungen (z.B. Transistor, Laser) wichtig ist." (Müller und Wiesner 2000, S. 1) (z. B. Zollman et al. 2002; Schneider und Meyn 2016; Reisch und Franz 2016)

Die SPS a ist häufig mit quantitativen Fragestellungen verknüpft. Es wird um Repräsentationen gerungen, die den formalen Apparat der Quantenphysik mit Schulmathematik handhabbar machen. Als Beispiel kann der Zeigerformalismus angeführt werden, welcher eine geometrische Repräsentation komplexer Zahlen bis hin zum Pfadintegral ist. Die Propagation wird durch eine Rotation des Zeigers dargestellt. Bei Superposition addieren sich die Zeiger vektoriell und das Quadrat der Länge des Zeigers lässt sich als Wahrscheinlichkeit für die Messung eines Quantenobjektes interpretieren.

Konzepte mit SPS b beschreiben vorwiegend das Verhalten von Quantensystemen oder Quantenobjekten. Hierzu werden meist Schlüsselexperimente der Quantenphysik wie der „Knallertest“ und der „Quantenradierer“ erläutert. Die Beschreibung der Einzelphotonenvarianten der Experimente durch die klassische Physik führt zu Widersprüchen und zeigt die Notwendigkeit einer neuen Theorie die Quantenphysik. Zum Beispiel wurden die Wesenszüge von Quantenobjekten (Küblbeck und Müller 2003) als ,,verbaler Formalismus" entwickelt, welcher die Beschreibung von Schlüsselexperimenten ermöglicht. Aber auch das Konzept von Fischler (1992) lässt sich hier einordnen. Dieses Konzept zeichnet sich dadurch aus, dass Lernerschwierigkeiten analysiert wurden und daraus resultierend Bezüge zur klassischen Physik vermieden werden. Quantenphänomene werden in diesem Zugang eindeutig von klassischen Phänomenen abgegrenzt.

Bei SPS c steht meist die Atomphysik im Vordergrund, hier vor allem der Übergang vom Bohrschen Atommodell zum quantenmechanischen Orbitalmodell. Besonders in frühen Konzepten zur Quantenphysik ist diese Schwerpunktsetzung häufig zu finden. Hierbei wird die physikalische Argumentation soweit verfeinert, dass eine Erklärung im Rahmen der klassischen Theorie versagt. Beispiel Bohrsches Atommodell: Elektronen auf einer Kreisbahn werden stets beschleunigt und emittieren nach der klassischen Elek- 
trodynamik Energie als elektromagnetische Strahlung. Ein solches System muss kollabieren. Quantenphysik wird bei dieser Schwerpunktsetzung präsentiert, um diesen Konflikt aufzulösen: Die Lösungen der Schrödingergleichung sind stationär.

Unter der SPS d wurden anfänglich Konzepte subsumiert, die den LASER, die Funktionsweise von Transistor und LED erklären (Zollman et al. 2002). In der jüngsten Zeit erfährt die SPS d zahlreiche neue Varianten, da Quantentechnologien ausgereifter sind und für das alltägliche Leben zunehmend relevanter werden (QUTEGA, Leuchs et al. 2017). Hierdurch verschiebt sich der Fokus hin zu Themen aus dem Bereich Quanteninformation (Quantencomputer, Quantenkryptographie usw.; siehe Schneider und Meyn 2016; Reisch und Franz 2016). Diese Schwerpunktsetzung nähert sich somit der SPS b an, die Betonung von Quantentechnologien in diesen Konzeptionen ist stärker als vorher auf den Begriff „Quanten“ fokussiert. Auf inhaltlicher Ebene kann ebenfalls differenziert werden. Traditionelle Konzepte begannen mit der klassischen Mechanik und führen hin zur Quantenmechanik. Dieser Weg kulminiert meist in einer Betrachtung des Wasserstoffatoms.

Bronner (2010) kritisiert an solchen Zugängen, dass dabei die Quantenoptik zu kurz kommt. Aus fachlicher Sicht lässt sich ein Zugang, der in der Optik startet und über die Quantenoptik hin zur Quantenelektrodynamik überleitet, kostengünstiger und nachhaltiger mit Realexperimenten anreichern (Bronner 2010; Scholz et al. 2018). Feynman (2006) unterstreichen zudem die Leistungsfähigkeit der Quantenelektrodynamik durch ihre Anwendung auf einfache Phänomene aus der Optik.

Zusätzlich unterscheiden sich die Konzepte auch im Umfang: Das Münchner Konzept zur Quantenphysik ist zum Beispiel thematisch sehr umfassend und geht weit über die klassischen Schulinhalte hinaus. Zudem wird das Konzept durch zahlreiche Materialien angereichert, die in der Schule ihren Einsatz finden können. Das Konzept von Rode (2013, 2017 ) beschreibt eine einzelne Unterrichtseinheit zur Quantenphysik und zeigt dabei Experimentiermöglichkeiten und den Einsatz von Zeigermodellierungen auf. Andere Konzepte beziehen sich auf einzelne Unterrichtseinheiten zu einem Thema innerhalb der Quantenphysik (zum Beispiel: Schneider und Meyn 2016; Reisch und Franz 2016).

Die Verankerung von Konzepten zur Quantenphysik in Curricula (vgl. Kultusministerkonferenz 2020a), Bildungsstandards (Kultusministerkonferenz 2020b) und Zentralprüfungen hängt von zahlreichen Faktoren ab: Neben den Qualitätskriterien der Konzepte, wie fachliche Relevanz, Angemessenheit und Anschlussfähigkeit, spielt auch die Zusammensetzung der Lehrplankommission eine wesentliche Rolle. Die Ausprägung der Lehrpläne/Curricula der verschiedenen Länder ist somit höchst unterschiedlich: Die optionale
Verankerung des Zeigerformalismus im niedersächsischen Kerncurriculum (Chrost et al. 2009) ist zum Beispiel eine besondere Rahmenbedingung, die in keinem anderen Bundesland zu finden ist. Weiterhin lässt sich in Niedersachsen eine inhaltliche Orientierung an den Wesenszügen erkennen. Auch in den Einheitlichen Prüfungsanforderungen (Kultusministerkonferenz 2004), einem länderübergreifenden Dokument, finden sich die Wesenszüge in leicht abgewandelter Form wieder. Das Experiment „Quantenradierer" ist explizit in den Anforderungen als Beispielaufgabe für eine schriftliche Abiturprüfung im Grundkurs angeführt. Diese Aufgabe wird unter anderem im Konzept von Brachner und Fichtner (1977) zur Demonstration des Fundamentalprinzips genutzt. Das Fundamentalprinzip (z.B. Feynman et al. 1963, S. 1-7), also die Komplementarität von Welcher-Weg-Information und Interferenz, spielt in den meisten Konzepten eine wichtige Rolle.

Zusammenfassend stehen Lehrende, die Quantenphysik in der Schule unterrichten, einer Vielzahl unterschiedlicher Konzepte gegenüber, welche sich nicht offensichtlich gegeneinander auszeichnen und sehr unterschiedliche Schwerpunktsetzungen aufweisen. Wie die subjektive Sicht der Lehrerinnen und Lehrer auf die vorhandenen Konzeptionen aussieht, ist dabei eine offene Frage. Schließlich sind sie es, die Schülerinnen und Schüler auf das Abitur vorbereiten und hierbei die Anforderungen aus den Curricula der Länder umsetzen.

\section{Die subjektive Sicht der Lehrenden auf Konzepte zur Quantenphysik}

Während Meta-Analysen zu den Konzepten in der Quantenphysik existieren (z. B. Bronner 2010; Burkard 2009; Müller 2005), gibt es keine empirische Basis für die subjektive Sicht der Lehrenden auf diese.

Bei der Erhebung der subjektiven Sicht der Lehrenden müssen folgende Rahmenbedingungen berücksichtigt werden:

1. Die potenziellen Teilnehmenden verfügen über stark unterschiedliches fachliches sowie didaktisches Wissen und einen unterschiedlichen Status in der eigenen Bezugsgruppe.

2. Eine theoriegeleitete Hypothesenbildung ist im zu bearbeitenden Themenfeld nicht möglich, da es nahezu unbearbeitet ist.

3. Aus Zeit- und Kostengründen können die Teilnehmenden nicht in physischen Treffen zusammengeführt werden.

Aus diesen Rahmenbedingungen ergeben sich spezifische Anforderungen an die Forschungsmethode. Als mögliche Methoden wurden Expertenbefragung (Gläser und Laudel 2010), Gruppendiskussion (Schäffer und Loos 2001) 
und Delphi-Methode (Häder 2009) identifiziert. Die DelphiMethode wurde hieraus ausgewählt, da sie gegenüber der isolierten Expertenbefragung einen Feedbackprozess bietet. Dieser ermöglicht ein intensives Überdenken und Bewerten der eigenen Aussagen und derer anderer Teilnehmenden. Gleichzeitig blendet die Methode gruppendynamische Effekte aus. Sie hebt sich hierdurch von der Gruppendiskussion ab und berücksichtigt in besonderem Maß die erste Rahmenbedingung. Die Delphi-Methode ist geeignet, um im Rahmen einer Studie Hypothesen zu generieren und zu verifizieren. Hierdurch wird dem Punkt zwei Rechnung getragen. Mittlerweile ist es auch etabliert, die Delphi-Methode online durchzuführen (vgl. Häder 2009), wodurch die Rahmenbedingung drei berücksichtigt wird.

Als ein Nachteil der Delphi-Methode ist die Auslegungsvielfalt der Methodik und, hiermit verbunden, eine unklare Formulierung anzuführen. Zu diesem Zweck wurde nach Häder (2009) im Vorfeld eine Typisierung der Delphi-Studie vorgenommen. Es handelt sich bei der vorgestellten Studie um eine Erhebung von Expertenmeinungen. Weber (2018) nimmt eine detailliertere Einordnung der Studie vor und stellt die hieraus resultierenden Implikationen dar. An dieser Stelle sollen die wichtigsten Charakteristika beschrieben werden.

\section{Beschreibung der Delphi-Methode}

Die Delphi-Methode ist eine rundenbasierte Kommunikationstechnik. In mindestens zwei Delphi-Runden wird einem Teilnehmer-Panel der Austausch über ein zuvor eingegrenztes Thema ermöglicht. Um gruppendynamische Effekte auszublenden, kommuniziert das Teilnehmer-Panel nicht direkt miteinander. Die Diskussionsleitung übernimmt das Monitoring-Team. Dieses stellt ein anonymes und kontrolliertes Feedback in Form eines Runden-Ergebnisses bereit und macht dieses in der nächsten Runde dem TeilnehmerPanel zugänglich, mit dem Ziel: ,,[...] den Einfluss psychologischer bzw. situativer Faktoren wie Überredung, Abneigung, den mitreißenden Einfluss einer Mehrheitsmeinung zu vermeiden“ (Ammon 2009, S. 459). Die Status-Inhomogenität im Teilnehmer-Panel kann somit durch die DelphiMethode handhabbar gemacht werden.

Bezeichnend für eine klassische Delphi-Runde sind fünf Bearbeitungsschritte (Häder 2009, S. 24; Ammon 2009, S. 460):

1. Operationalisierung der zu bearbeitenden Frage-/Problemstellung

2. Design eines standardisierten und formalisierten Frageprogramms

3. Bearbeitung des Frageprogramms durch das TeilnehmerPanel
4. Feedbackprozess durch das Monitoring-Team

a. Anonymisierung der Einzelantworten

b. Ermittlung des Runden-Ergebnisses aus Antworten und gegebenen Begründungen

c. Information des Experten-Panels über das Runden-Ergebnis

5. (Mehrfache) Wiederholung des Verfahrens bis ein $\mathrm{Ab}$ bruchkriterium erfüllt wurde.

In der physikdidaktischen Forschung wurde die DelphiMethode mehrfach eingesetzt und konnte starke Evidenzen hervorbringen:

- Physikalische Bildung: Eine curriculare Delphi-Studie (Häussler et al. 1980)

- Ziele, die Lehrende mit dem Experimentieren in der naturwissenschaftlichen Ausbildung verbinden (Welzel et al. 1998)

- Quantenphysik in der Schule: Bestandsaufnahme, Perspektiven und Weiterentwicklungsmöglichkeiten durch die Implementierung eines Medienservers (Burkard 2009)

Das Teilnehmer-Panel der aufgeführten Studien wurde selektiert. Die Auswahl der Teilnehmenden erfolgte dabei mit Hilfe von definierten Kriterien und teilweise mit Einladungssystem, d.h. Teilnehmende können selbst ,passende Personen“" zur Teilnahme an der Studie einladen (z. B. Burkard 2009).

\section{Eckdaten der Delphi-Studie}

Die hier beschriebene Delphi-Studie fand im Zeitraum vom 22.05.2014 bis zum 31.07.2015 statt und umfasst drei Stufen. Vorab wurde eine Pilotstudie mit Fachleitern durchgeführt, um den Fragebogen der ersten Runde zu entwickeln. An die Teilnehmenden der Hauptrunden wurde die Anforderung gestellt, dass diese mindestens einmal das Thema Quantenphysik in der Schule unterrichtet haben. Ein späterer Einstieg war zudem möglich: Eine Teilnahme an der dritten Runde war zum Beispiel möglich, ohne an einer der vorherigen Runden teilgenommen zu haben. Der Teilnehmerpool ist somit weitestgehend offengehalten. Die Studie unterscheidet sich hierdurch von den zuvor benannten Delphi-Studien aus der physikdidaktischen Forschung.

Zur Akquirierung der Teilnehmenden wurden sämtliche Schulen mit gymnasialer Oberstufe in Niedersachsen angeschrieben. Die Stufe 1 startet mit 84 Teilnehmenden. 35 Teilnehmende hiervon gehen in die Stufe 2 über. 18 Teilnehmende nahmen an der zweiten Stufe teil, ohne an Stufe 1 teilgenommen zu haben. Es ergeben sich 53 Teilnehmende in der zweiten Stufe. Hiervon gehen 39 Teilnehmende in die dritte Stufe über. 14 Teilnehmende nahmen an der ersten und dritten Stufe teil, 22 Teilnehmende nahmen ausschließ- 
lich an der dritten Stufe teil. Die Anzahl der Teilnehmenden in der dritten Runde steigt somit gegenüber der zweiten Runde an auf 75 Teilnehmende. Mit 26 Teilnehmenden nahmen über $30 \%$ der ersten Stufe auch an den Folgestufen teil. Die beobachtete Panelmortalität ist bei Delphi-Studien üblich (vgl. Häder 2009). Hinzu kommt der Einbruch der Teilnehmendenanzahl in der zweiten Stufe, welcher durch den Zeitpunkt (Jahreswechsel) und den erhöhten Bearbeitungsumfang zu erklären ist.

Um einen möglichst authentischen Eindruck von der subjektiven Sicht der Lehrenden zu erhalten, wurde darauf geachtet, dass sowohl erfahrene Lehrende als auch Anfänger im Unterrichten von Quantenphysik im Teilnehmerpool vertreten sind. Dies wurde durch gezielte Reaktivierung der Teilnehmenden erreicht. Dabei ist zu unterstreichen, dass unter den erfahrenen Lehrenden auch Fachleitende und LehrerInnenausbildende teilgenommen haben. Als Indikator für die Erfahrung wurde die Anzahl der unterrichteten Oberstufenkurse ausgewählt.

Abb. 2 stellt die Zusammensetzung des Teilnehmerpools dar. Die größte Gruppe bilden die Teilnehmenden mit mehr als sechs unterrichteten Oberstufenkursen: Dies sind die erfahrenen Teilnehmer der Studie. Am schwächsten im Pool vertreten sind die Beginner im Unterrichten von Quantenphysik. Der Anteil der Anfänger im Pool ist dennoch in allen Runden größer als 10\%. Das Monitoring-Team achtet im Delphi-Prozess darauf, dass auch die Interessen der Anfänger wahrgenommen und im Panel diskutiert werden.
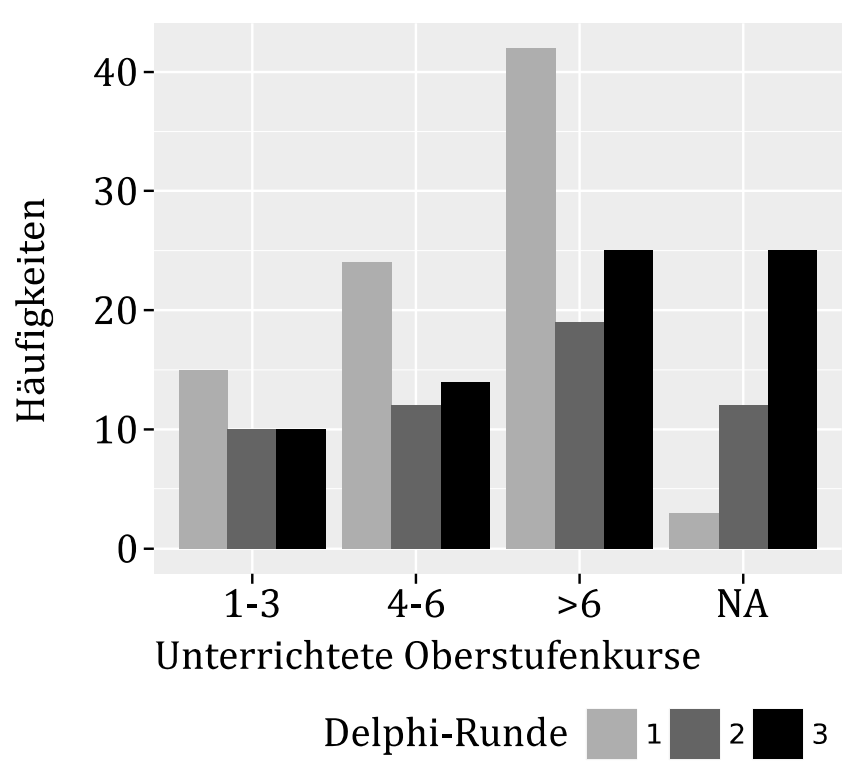

Abb. 2 Zusammensetzung des Expertenpanels

\section{Exemplarisches Vorgehen von der ersten bis zur letzten Runde}

Von besonderer Bedeutung bei der Durchführung einer Delphi-Studie ist die Arbeit des Monitoring-Teams. Das Team hat die Aufgabe, das Kondensat der Runden zu extrahieren und greift somit moderierend in den methodischen Prozess ein. In einem explorativen qualitativen Ansatz wurden dabei die Antworten der Teilnehmenden gebündelt und so gemeinsame Facetten herausgearbeitet, welche die Grundlage für die folgende Delphi-Runde darstellen. Um an dieser Stelle einen Einblick in die Arbeit des Monitoringteams $\mathrm{zu}$ geben, wird hier ein exemplarisches Vorgehen von der ersten bis zur letzten Runde vorgestellt. Hierfür wird die Entwicklung einer zentralen Frage über die verschiedenen Runden hinweg dargestellt und Antworten der Teilnehmenden aufgezeigt, die Aussagenbündel repräsentieren, welche maßgeblich den weiteren Verlauf der Studie beeinflussen.

In der ersten Delphi-Runde wurde nachstehende Frage an die Teilnehmenden gerichtet:

Das Thema ... aus der Quantenphysik ist/war für meine Schülerinnen und Schüler eine besondere Herausforderung, denn ... Das hat geholfen/würde ich mir wünschen:

Die beiden folgenden Antworten stehen prototypisch für viele im Antwortpool:

„Potenzialtopf, Farbstoff, Komplementarität, Schrödingergleichung; Alle Themen sind $\mathrm{m}$. W. nur theoretisch erarbeitbar und man kann sie einfach nur glauben und auswendig lernen." (Antwort 0)

„Der ,,andere“ Gedankenansatz, dass sich Quantenobjekte anders als klassische Objekte verhalten und wir eine genaue Aussage über das Verhalten der Quantenobjekte erst unter bestimmten Bedingungen machen können [...], machte große Probleme. Nur die sehr sehr leistungsstarken Schüler nutzen dieses Angebot zu Diskussionen.“ (Antwort 1)

Aus der ersten Antwort lässt sich ableiten, dass die Inhalte nicht in einem erkenntnistheoretischen Prozess erarbeitet werden können, sondern rezipiert werden. Dies wird der Sachstruktur der Themen zugeschrieben. Als Erklärung hierfür ist das Fehlen von Experimenten im Schulunterricht denkbar. Der Begriff Komplementarität ist unter den Wesenszügen der Quantenphysik zu finden (Küblbeck und Müller 2003). Die zweite Antwort beschreibt Quantenobjekte und den Messprozess, welche ebenfalls Teil der Wesenszüge sind. Zudem wird betont, dass nur die leistungsstarken Schülerinnen und Schüler an den Diskussionen teilhaben. Auf der Grundlage dieser und weiterer Antworten wurde für die zweite Runde eine Paraphrase (nachstehend 
fett) erstellt, die auf die gesamten Wesenszüge generalisiert wurde:

\section{Ein Großteil der Wesenszüge ist nur theoretisch zu erarbeiten; man kann diese einfach nur glauben und auswendig lernen.}

Welche Bedeutung hat obenstehende Paraphrase für

Ihren Unterricht und wie bewerten Sie in diesem $\mathrm{Zu}$ -

sammenhang den Einsatz von Animationen, Simula-

tionen und Originalmesswerten?

Mit dem gegebenen Arbeitsauftrag wird der Fokus auf Möglichkeiten gelenkt, die eine Alternative zu Realexperimenten im Unterricht sein können.

Repräsentativ für ein Aussagenbündel steht die Antwort eines Teilnehmenden:

"Quantenphysik ist ein sehr theoretisches Gebiet in der Physik und oft nicht schnell nachweisbar. Eine Simulation hilft es zu verbildlichen, jedoch ist der Nachteil dabei, dass die Simulation eben etwas „Gemachtes" ist, was etwas zeigt, jedoch auch den Programmierkünsten des Entwicklers zugrunde liegt. Ich kann auch eine Lüge simulieren.“ (Antwort 2)

Die Antwort verdeutlicht, dass Simulationen im Unterricht als hilfreich empfunden werden.

Als möglicher Nachteil wird hervorgehoben, dass Simulationen nicht zwangsweise das Verhalten eines physikalischen Systems beschreiben müssen. Dieses Argument wurde in der dritten Runde aufgegriffen. Es sollte insbesondere geklärt werden, ob auch andere Lehrende diese kritische Position bei den Lernenden wahrnehmen.

Bewerten Sie folgende Aussage: Meine Schüler haben kein Vertrauen in die Ergebnisse von Simulationen im Bereich der Quantenphysik.

Mit Hilfe einer Fünf-Punkt-Likert-Skala konnten die Teilnehmenden der dritten Runde die Aussage bewerten. Die

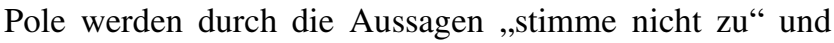
„stimme zu“ gebildet. Zur Darstellung der Ergebnisse werden „Diverging Stacked Bar Charts“ verwendet (Abb. 3 erstellt nach Bryer und Speerschneider 2016).

Robbins und Heiberger (2011) erläutern diese Form der Darstellung und grenzen diese gegenüber anderen Darstel- lungen von likert-skalierten Items ab. Die Breite des gestapelten Balkens entspricht $100 \%$. Der Balken ist relativ zur Skalenmitte ausgerichtet. Hierdurch wird Zustimmung und nicht Zustimmung in Ausschlägen nach rechts beziehungsweise links dargestellt. Die drei Prozentzahlen repräsentieren die Anteile derer, die nicht zustimmen (76\%), neutral stimmen (16\%) beziehungsweise zustimmen (7\%). Abweichungen in der Summe von $100 \%$ entstehen durch Rundung.

Der Median wurde als quantitative Repräsentation der Gruppenmeinung gewählt. Dieser ist im obigen Beispiel als Antwort „stimme eher nicht zu“ zu werten. Lehrende nehmen eine Skepsis gegenüber Simulationen bei den Schülerinnen und Schülern also nicht wahr.

\section{Repräsentation der qualitativen Daten mittels Wordcloud}

Als Analysewerkzeug für die freien Antworten wurden Wordclouds ausgewählt (vgl. Abb. 7), denn diese ermöglichen eine visuelle qualitative Einordnung der Nennungen eines Schlüsselbegriffs. Sie helfen somit, die Relevanz der Antworten im gesamten Antwortfeld einzuschätzen.

Die Textgröße skaliert mit der Häufigkeit der Nennungen. Horizontale und vertikale Ausrichtung der Wörter sowie deren Positionierung sind zufällig. Den Häufigkeiten liegt im Folgenden eine Zuordnung zu einem Schlüsselwortsystem zu Grunde.

Dieses System wurde aus den Antworten konstruiert (explorativer Ansatz) und anschließend zweifach kodiert. Cohens-Kappa dient als Quantität für die Intercoder-Reliabilität. Es wird die weit gebräuchliche Interpretation nach Landis und Koch (1977) verwendet.

\section{Ergebnisse}

Nachfolgend entlang der Leitfragen aus dem Abschnitt Ziele und Forschungsfragen ausgeführt; die Ergebnissdarstellung folgt damit nicht der Chronologie der Datengewinnung innerhalb der Delphi-Runden.
Abb. 3 Antwort aus der dritten Delphi-Runde: Lernende haben Vertrauen in Simulationen (Weber 2018, S. 52)

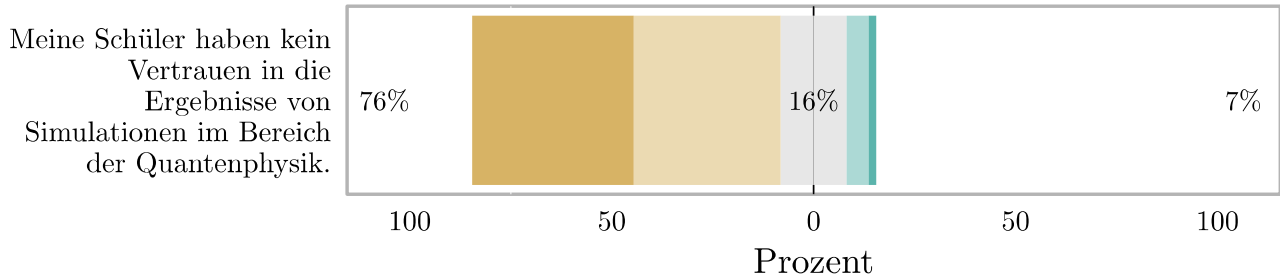

$\begin{array}{lllll}\text { Stimme nicht zu } & \text { Stimme eher nicht zu Neutral } & \text { Stimme eher zu } & \text { Stimme zu }\end{array}$ 


\section{Welche fachdidaktischen Konzepte werden von Lehrkräften bei der Planung und Durchführung von Unterricht in der Quantenphysik berücksichtigt?}

In der zweiten Delphi-Runde wurde den Teilnehmenden der Auftrag gegeben:

Bitte markieren Sie im Folgenden, aus welchen Konzepten sie Bestandteile in Ihren Unterricht einfließen lassen (gA/eA) und welche Konzepte Ihnen nicht bekannt sind.

Bei der Beantwortung der Frage konnte nach erhöhtem Anforderungsniveau (eA) und grundlegendem Anforderungsniveau (gA) differenziert werden. Weiterhin konnte von den Teilnehmenden angegeben werden, dass ihnen ein Konzept unbekannt ist. Wurde keine der zur Verfügung stehenden Optionen angewählt, zählen diese Antworten als NA (not available).
Zur Auswahl standen Lehrbuchkonzepte und Konzepte aus der Fachdidaktik.

Tab. 1 stellt die Ergebnisse dar: Konzepte aus der Fachdidaktik sind weitgehend unbekannt. Ausnahmen hiervon sind das Münchner Konzept (Müller und Wiesner 2000) und die Wesenszüge der Quantenphysik (Küblbeck und Müller 2003). Die Lehrbuchkonzepte und die Konzepte mit Zeigerformalismus sind durchweg bekannt und fließen sowohl auf erhöhtem als auch auf grundlegendem Anforderungsniveau ein.

Die Frage zur Berücksichtigung von Konzepten wurde als Einleitung dem folgenden offenen Arbeitsauftrag vorangestellt:

Bitte beschreiben Sie Konzeptbestandteile, von denen Ihr Unterricht im besonderen Maße profitiert.

30 von 53 Teilnehmende bearbeiteten diesen Auftrag und stellen insbesondere heraus, dass in der Praxis eine Mischung aus den verfügbaren Konzepten unterrichtet werde.
Tab. 1 Wahrnehmung von Konzepten zur Quantenphysik
Abb. 4 Die Rolle der Wesenszüge im Unterricht (DiskuWesz Diskussion der Wesenszüge im Unterricht, RoFWesz Wesenszüge als roter Faden im Unterricht, $e A$ erhöhtes Anforderungsniveau, $g A$ grundlegendes Anforderungsniveau; die genaue Itemformulierung kann dem Anhang entnommen werden)

\begin{tabular}{lllll}
\hline Konzept & gA & eA & Unbekannt & NA \\
\hline $\begin{array}{l}\text { Münchner Konzept und milq } \\
\text { (Müller und Wiesner 2000) }\end{array}$ & 20 & 29 & 17 & 4 \\
$\begin{array}{l}\text { Erlanger Konzept } \\
\text { (Bronner et al. 2009) }\end{array}$ & 2 & 1 & 46 & 5 \\
$\begin{array}{l}\text { Bremer Konzept } \\
\text { (Niedderer 1992) }\end{array}$ & 1 & 2 & 46 & 5 \\
$\begin{array}{l}\text { Berliner Konzept } \\
\text { (Fischler und Lichtfeldt 1992) }\end{array}$ & 1 & 2 & 47 & 4 \\
$\begin{array}{l}\text { Konzept von Brachner und Fichtner } \\
\text { (Brachner und Fichtner 1977) }\end{array}$ & 1 & 1 & 43 & 8 \\
$\begin{array}{l}\text { Konzepte mit Zeigerformalismus } \\
\text { (Küblbeck und Müller 2003; Bader 2000; Erb und Schön 1997) }\end{array}$ & 27 & 36 & 6 & 6 \\
$\begin{array}{l}\text { Wesenszüge der Quantenphysik } \\
\text { (Küblbeck und Müller 2003) }\end{array}$ & 15 & 24 & 19 & 5 \\
$\begin{array}{l}\text { Visual Quantum Mechanics } \\
\text { (Zollman et al. 2002) }\end{array}$ & 1 & 3 & 40 & 10 \\
$\begin{array}{l}\text { Lehrbuchkonzept: Dorn-Bader } \\
\text { Lehrbuchkonzept: Metzler } \\
\text { Lehrbuchkonzept: Impulse }\end{array}$ & 28 & 36 & 3 & 9 \\
\hline
\end{tabular}

$g A$ Grundlegendes Anforderungsniveau, $e A$ Erhöhtes Anforderungsniveau, NA keine Auswahl getroffen

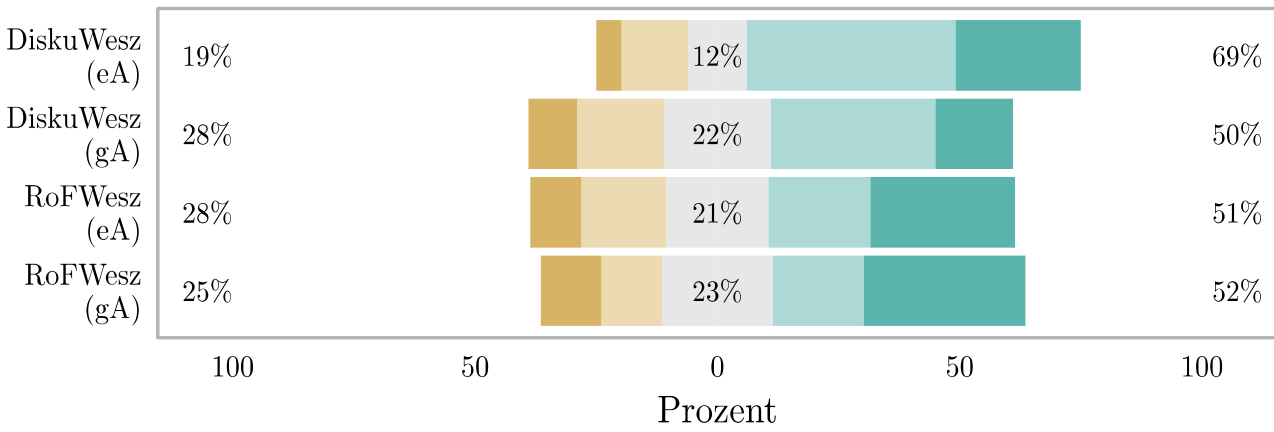

Stimme nicht zu Stimme eher nicht zu Neutral $\quad$ Stimme eher zu Stimme zu 
„[...] Dieses Konzept [Müller/Wiesner] angereichert mit den Darstellungen vom Dorn-Bader und Impulse bietet eine Grundlage für meinen Unterricht. [...]“ (Antwort 3)

Weiterhin wird benannt, dass bestehende Konzepte durch Realexperimente unterstützt werden sollten, wobei wenige im Schulunterricht durchführbare Experimente existieren. Der Zeigerformalismus und die Wesenszüge werden mehrfach als tragfähige Konzepte herausgestellt. Die Unterrichtsmaterialien des Münchner Konzepts werden dabei als hilfreich benannt. Auch populärwissenschaftliche Bücher wie Feynmans QED (2006) fließen in den Unterricht ein.

Eine starke Orientierung am Kerncurriculum (Chrost et al. 2009) wird von den Befragten herausgestellt:

„Der Unterricht orientiert sich stark am $\mathrm{KeCu}$ [Kerncurriculum], es gibt keinen „roten Faden“ hinsichtlich eines ausgearbeiteten Konzeptes. Hier besteht noch Handlungsbedarf [...].“ (Antwort 4)

Die freien Antworten der Teilnehmenden unterstreichen zusätzlich die herausgehobene Rolle der Wesenszüge in der Vermittlungspraxis von Quantenphysik. Die quantitativen Antworten aus der dritten Runde geben einen Einblick, wie das gesamte Expertenpanel die Rolle der Wesenszüge einschätzt (siehe Abb. 4).

Die ausgewählten Items stellen heraus, dass die Wesenszüge Diskussionen im Unterricht ermöglichen DiskuWesz (Zustimmung eA: 69\%/gA: 50\%). Weiter nehmen die Wesenszüge eine strukturelle Funktion ein, indem sie sich wie ein roter Faden durch den Unterricht ziehen, RoFWesz (Zustimmung eA: $51 \% / \mathrm{gA}: 52 \%$ ).

Beide Items bestätigen die Relevanz und Akzeptanz der Wesenszüge durch die Lehrenden. DiskuWesz unterstreicht mit dem Unterschied zwischen eA und gA zudem, dass vor- wiegend leistungsstarke Schülerinnen und Schüler an den Diskussionen teilhaben.

\section{Welle-Teilchen-Dualismus}

Eine Gemeinsamkeit moderner Konzepte in der Quantenphysik ist die Vermeidung eines naiven Dualismus-Begriffs. Folgende Formulierungen werden zum Beispiel vermieden: ,Je nach experimenteller Fragestellung verhält sich das Quantenobjekt mal als Welle und mal als Teilchen.“ Genauso wie: „Ein Quantenobjekt ist zugleich Welle und Teilchen." Derartige Formulierungen stammen meist aus den Anfängen der Quantenphysik und suggerieren eine Beliebigkeit der Natur. Über diesen Stand ist die fachliche Forschung weit hinaus. Weiter wird hierdurch eine Ableitung der Quantenphysik aus der klassischen Physik impliziert, die aber nicht möglich ist. Andererseits ist der WelleTeilchen-Dualismus unter den Top-Themen, die durch mindestens $90 \%$ der Teilnehmenden als unverzichtbar für eine intellektuell redliche Auseinandersetzung mit dem Themenfeld Quantenphysik identifiziert wurden (Abb. 5).

Eine offene Frage aus der zweiten Runde ermöglicht einen detaillierteren Einblick in die Sicht der Lehrenden auf den Dualismus-Begriff:

Auch eine Dualismus-Debatte prägte das Antwortenfeld. Ich möchte Sie darum bitten, die Rolle des WelleTeilchen-Dualismus in Ihrem Unterricht zu beschreiben.

Die kontroversen Beiträge lassen sich in zwei Gruppen einteilen. Befragte mit Beiträgen zur ersten Gruppe empfinden den Dualismus als hilfreich für den Unterricht und beschreiben diesen als Grundgedanken und Merkmal der Quantenphysik. Der Einstieg über den Photoeffekt und die Elektronenbeugungsröhre wird dabei zur Erzeugung eines
Abb. 5 Themen, die von über $90 \%$ der Teilnehmenden für eine intellektuell redliche Auseinandersetzung mit der Quantenphysik als unverzichtbar bewertet wurden

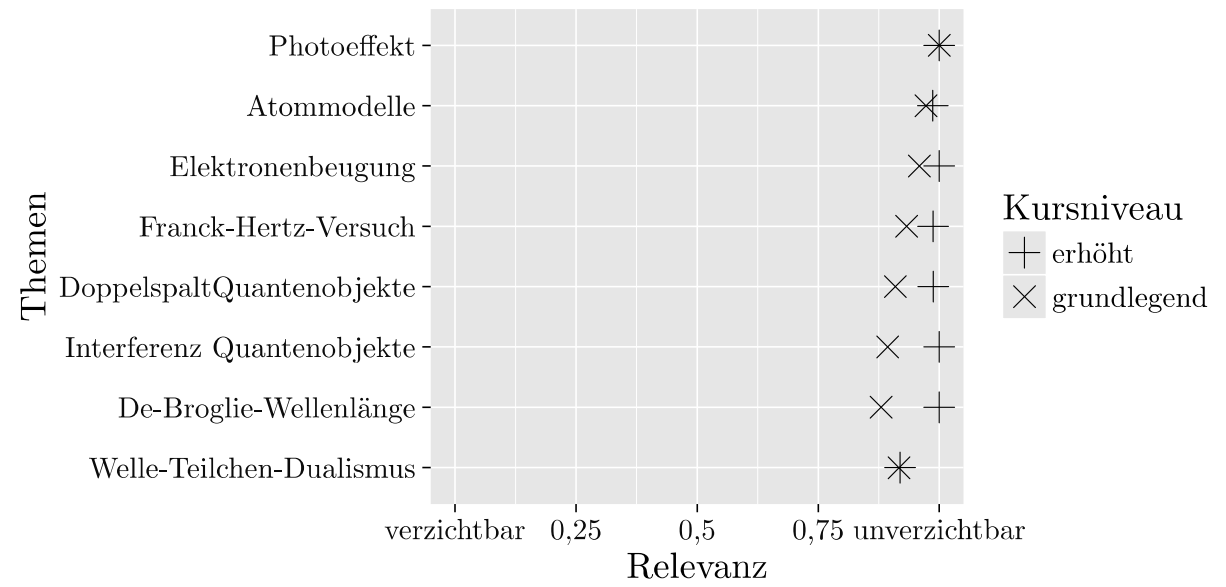


Abb. 6 Die Sicht auf den Dualismusbegriff in der Unterrichtspraxis (ModBi Dualismusbegriff unterstreicht den Modellbildungscharakter, DiskSpFi Empfindung der Diskussion um den Dualismus-Begriff als spitzfindig, ObjErfW problematisch, Dualismus legt Quant als Objekt aus der Erfahrungswelt der Schüler nahe, $e A$ erhöhtes Anforderungsniveau, $g A$ grundlegendes Anforderungsniveau; die genaue Itemformulierung kann dem Anhang entnommen werden)

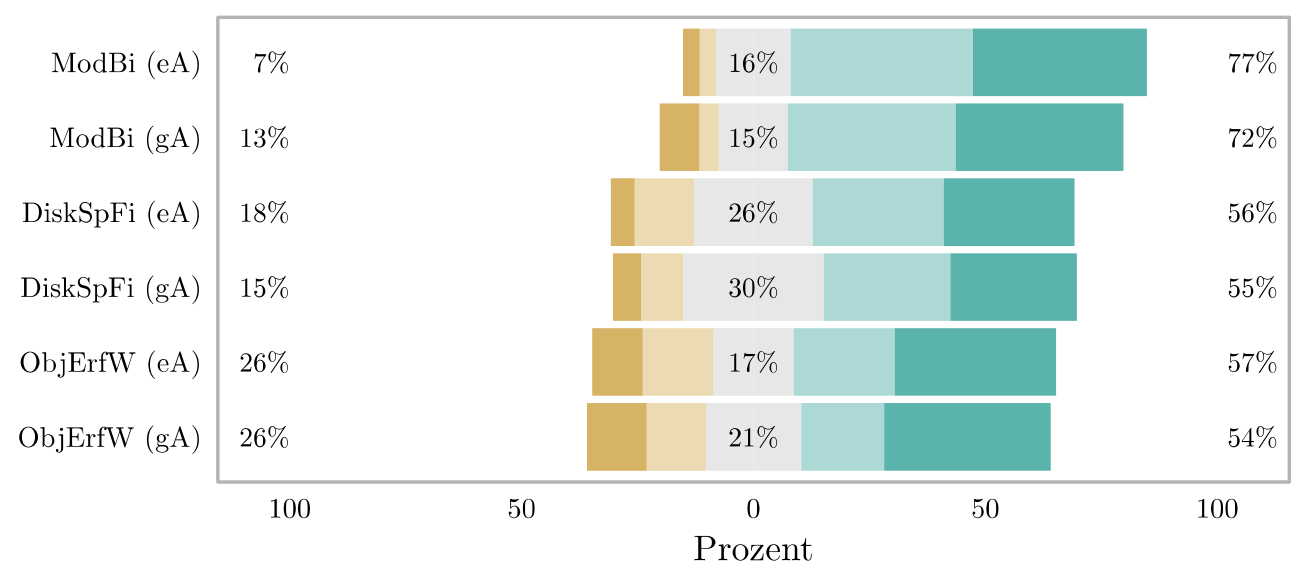

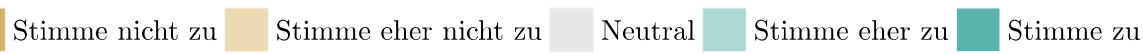

kognitiven Konflikts genutzt, der anschließend durch die Einführung des Dualismus aufgelöst wird. Auch auf den Modellbildungscharakter wird hierbei verwiesen. Je nach Situation wird das System unterschiedlich modelliert.

Der Welle-Teilchen-Dualismus wird zur Verdeutlichung des modellbildenden Charakters der Wissenschaft Physik genutzt:

,[...] Macht sich der Mensch an der Stelle nicht nur ein Modell und zieht mit seiner begrenzten Auffassungsgabe dann eine geeignete Karte?"“(Antwort 5)

Befragte mit Beiträgen zur zweiten Gruppe vermeiden oder problematisieren den Welle-Teilchen-Dualismus im Unterricht. Es werden Quantenobjekte als neues Konzept eingeführt. Die Eigenschaften dieser neuen Klasse von Objekten werden durch die Wesenszüge der Quantenphysik beschrieben:

„Ich versuche den Begriff im Unterricht zu vermeiden und gerade durch das Konzept Quantenobjekt + Wesenszüge zu ersetzen. Aber oftmals wird dieser Begriff von den Schülern selbst in den Unterricht hineingetragen und muss entsprechend problematisiert werden." (Antwort 6)

Im zweiten Teil der Antwort 6 wird darauf hingewiesen, dass der Dualismus-Begriff außerhalb von didaktischen Diskussionen durchaus gebräuchlich ist. Dies stellt keine Einzelmeinung dar. Einer der Teilnehmenden beschreibt, dass Fachphysiker keine Probleme mit dem Begriff haben.

,[...] In der didaktischen Literatur wird er [der Dualismusbegriff] ja vielfach vermieden. Mein Eindruck ist, dass er im universitären Bereich durchaus ,hemdsärmeliger" verwendet wird." (Antwort 7)

Die Haltung gegenüber dem naiven Dualismus-Begriff ist in der Schulpraxis nicht so eindeutig wie es in der didaktischen Literatur der Fall ist.
Eine Auswahl von Items aus der letzten Runde zum Dualismus-Begriff unterstreicht die kontroversen Ansichten der Lehrenden (Abb. 6).

Die Diskussion um den Dualismus-Begriff wird von über der Hälfte der Befragten als spitzfindig empfunden (Disk$\mathrm{SpFi}$, Zustimmung eA: 56\%/gA: 55\%). Gleichzeitig erachtet die Hälfte des Panels es als problematisch, dass der Dualismus-Begriff die Vorstellung von einem ,Objekt“ aus der Erfahrungswelt der Lernenden nahelegt (ObjErfW, Zustimmung eA:57\%/gA: $54 \%$ ). Hierin zeigt sich die starke Heterogenität bezüglich der Einstellung zum Dualismus-Begriff. Einigkeit zeigt sich darin, dass der Dualismus-Begriff als gutes Beispiel für die Rolle von Modellbildung in der Physik erachtet wird (ModBi, Zustimmung eA: 77\%/gA: $72 \%)$.

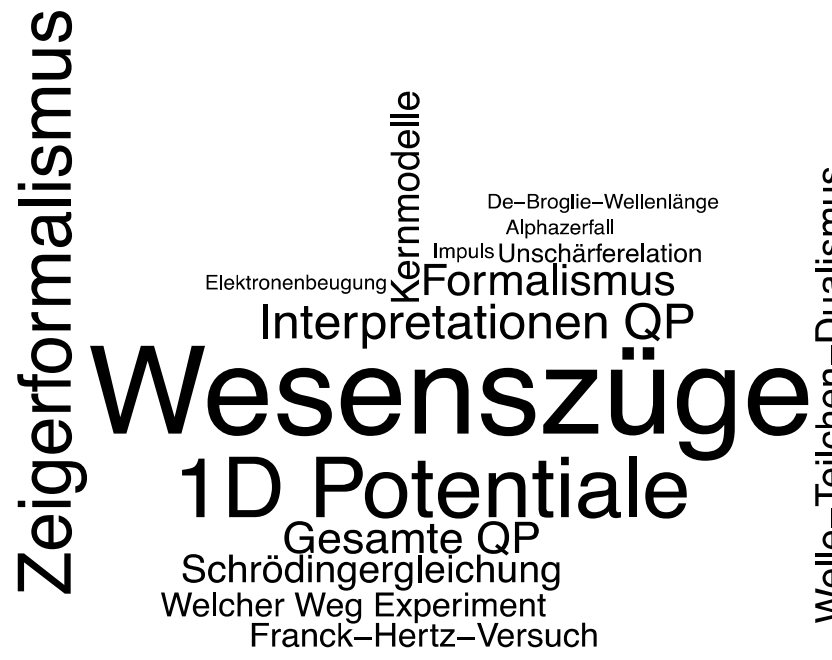

Abb. 7 Wordcloud zu den Themen der Quantenphysik, die als besondere Herausforderung für Schülerinnen und Schüler wahrgenommen werden 
Abb. 8 Umsetzung der Wesenszüge im Unterricht (RExpWesz Forderung, Realexperimente bei der Vermittlung der Wesenszüge, SimuWesz Verfügbare Simulationen veranschaulichen die Wesenszüge gut, RelImAbitur die Relevanz der Wesenszüge im Abitur ist im Verhältnis zum Aufwand zu gering, $e A$ erhöhtes Anforderungsniveau, $g A$ grundlegendes Anforderungsniveau; die genaue Itemformulierung kann dem Anhang entnommen werden)

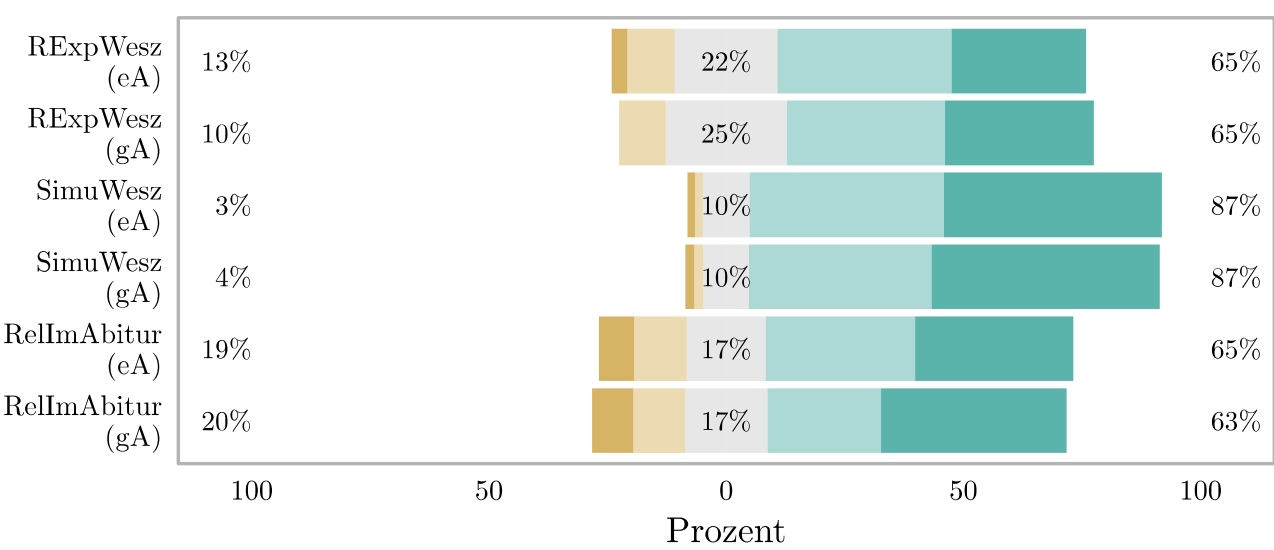

\begin{tabular}{ll|ll} 
Stimme nicht zu & Stimme eher nicht zu Neutral & Stimme eher zu & Stimme zu
\end{tabular}

\section{Besondere Herausforderungen für die Lernenden im Bereich der Quantenphysik}

In der ersten Delphi-Runde wurden Themen gesammelt, die in der Wahrnehmung der Lehrenden als besondere Herausforderung für die Schülerinnen und Schüler gelten. In Abb. 7 werden die Nennungen als Wordcloud dargestellt. Für die Zuordnung zum verwendeten Schlüsselwortsystem ergab sich ein Cohens-Kappa von 0,70 (Substantial) für die Übereinstimmung der zwei Coder. Zu den abgebildeten Nennungen lassen sich vier Überkategorien identifizieren: Atomphysik, Quantenobjekte, Formalismus und Interpretationen der Quantenphysik.

Auch die subjektiv empfundenen Herausforderungen beim Unterrichten von Quantenphysik wurden erhoben. Hierin enthalten sind die Äußerungen zu den Begriffen Wesenszüge, Welle-Teilchen-Dualismus, Elektronenbeugung, Welcher-Weg-Experiment, Unschärferelation und De-Broglie-Wellenlänge. $23 \%$ aller Zuordnungen entfielen auf den Begriff Wesenszüge der Quantenphysik. Dabei wurden vor allem die Begriffe Nichtlokalität und Komplementarität als schwierige Themen hervorgehoben und die Versuche „Quantenradierer“ und „Knallertest“ als WelcherWeg-Experimente benannt.

„Die [W]elcher-Weg-Experimente; Die Schüler haben besonders bei Elektronen Schwierigkeiten mit der Vorstellung. Ich würde mir eine gute (nicht so kindliche) Darstellung der Vorgänge als Animation wünschen." (Antwort 8)

Weitere Beiträge bestätigen, dass die Arbeit mit Simulationen ein wichtiger Bestandteil ihres Unterrichts ist. Insgesamt wird als besondere Herausforderung in diesem Themengebiet eine fehlende Anschauung angeführt.
„[...] Weiteres Problem: die Unmö[g]lichkeit der ,gegenständlichen“"Verbildlichung. (also: keine Bahnen, keine Kugeln etc.) [...]“ (Antwort 9)

In der zweiten Runde ergänzen Teilnehmende, dass hierdurch ebenfalls die Möglichkeit entstehe, auf Modelle und deren Rolle in der modernen Physik einzugehen. Einer der Teilnehmenden trifft die Aussage, dass es für die Abiturprüfungen reiche, zentrale Experimente und deren Interpretation auswendig zu lernen. Ein anderer erklärt, dass in dem Bereich aus zeitlichen Gründen viel auswendig gelernt werde.

Die dritte Runde zeigt eine klare Forderung nach Realexperimenten, welche die Wesenszüge veranschaulichen (Abb. 8, RExpWesz, Zustimmung eA: 65\%/gA: 65\%). Zudem wird auch herausgestellt, dass verfügbare Simulationen die Wesenszüge gut veranschaulichen (Abb. 8, SimuWesz, Zustimmung eA: $87 \% / \mathrm{gA}: 87 \%$ ) und unterstrichen, dass der Zeitaufwand für die Erarbeitung der Wesenszüge erheblich ist und in keinem Verhältnis zu deren Relevanz in den zentralen Abiturprüfungen steht (Abb. 8, RelImAbitur, Zustimmung eA: $65 \% / g A: 63 \%$ ).

\section{Wie wird die retrospektive Sicht auf die eigene Ausbildung im Themengebiet Quantenphysik beschrieben?}

50 Teilnehmende der ersten Runde machten Aussagen zur eigenen Ausbildung im Bereich Quantenphysik. Abb. 9 zeigt eine Übersicht der Themen, die in der Retrospektive der Teilnehmenden hätten umfassender thematisiert werden müssen. Insgesamt wird bemängelt, dass zu wenig didaktische Auseinandersetzung mit dem Themengebiet in der Ausbildung erfolgt, wobei die fachliche Ausbildung als umfänglich beschrieben wird. Begriffe wie Komplementarität, Verschränkung und Nichtlokalität - allesamt Wesenszüge, die bezeichnend für das Verhalten von Quantenobjekten 


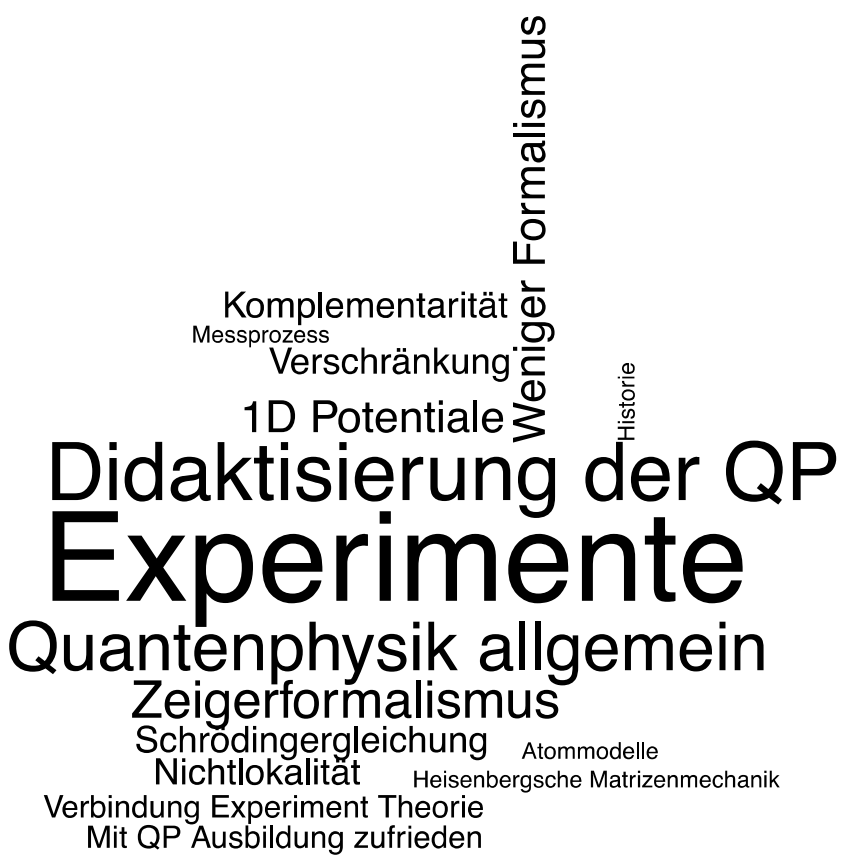

Abb. 9 Retrospektiv hätten diese Themen umfassender thematisiert werden müssen

sind - hätten laut den Lehrenden ebenfalls umfassender thematisiert werden müssen. Gerade diese Begriffe verdeutlichen die Modernisierung der Lehrpläne im Themenbereich der Quantenphysik (vgl. Pospiech und Schöne 2012). Laut den Teilnehmenden existiert eine Diskrepanz zwischen dem an der Universität gelehrten Inhalten und den im Unterricht benötigten Inhalten.

Studierende nehmen zu oft nur an Fachveranstaltungen teil und „rechnen“ dabei viel (zu viel). Sie können meiner Erfahrung nach dann oft noch nicht ein[ma]l in wenigen Sätzen den Kern erklären. Dies ist ein fachdidaktisches Problem, da in der Schule nicht (bzw. kaum) gerechnet wird, sondern im Wesentlichen erklärt wird. (Antwort 10)

Teilnehmende fühlten sich nach dem Studium nicht in der Lage, die an der Universität behandelten Inhalte für den Schulunterricht angemessen zu elementarisieren. Als Grund hierfür wurde eine zu starke Fokussierung auf das formale Kalkül der Quantenphysik benannt.

Andere Teilnehmende beschreiben, dass sie eine bessere Verknüpfung zwischen Theorie und Experimenten gewünscht hätten. Der größte Teil der Antworten $(23 \%$ der Zuordnungen) bezog sich auf Experimente: Die Teilnehmenden fordern mehr Experimente, die begleitend zu den Vorlesungen durchgeführt werden. Benannt wurden in diesem Zusammenhang Experimente, welche die Wesenszüge herausstellen, wie zum Beispiel Versuche mit dem Doppelspalt und Welcher-Weg-Experimente.

\section{Konstruktion der Leitlinien für die Lehrerfortbildung}

Die beschriebene Delphi-Studie ermöglicht die wechselseitige Beziehung zwischen Konzeptionen und der Sicht der Lehrenden auf diese. Im nächsten Schritt werden auf dieser Basis Leitlinien für Lehrerfortbildungen über Quantenphysik konstruiert. Dieser Prozess wurde ebenfalls im Rahmen der Delphi-Studie bereits mit angelegt. So wurden mit entsprechenden Items Daten zum persönlichen Interesse und zur Rahmengestaltung von Fortbildungen erhoben.

Herausgearbeitet wurden folgende Leitlinien (Weber 2018, S. 146):

1. Schlüsselexperimente zur Quantenphysik sollten Teil der Fortbildung sein.

2. Für die Beschreibung von Schlüsselexperimenten ist der Bezug zu den Wesenszügen der Quantenphysik zentral.

3. Im Unterricht einsetzbare Originalmesswerte, Simulationen und Animationen sollten die Schlüsselexperimente ergänzen.

4. Bei der Erklärung von Schlüsselexperimenten wird der Dualismus-Begriff als Versagen der klassischen Modellbildung herausgestellt. Als Quantenmodell treten die Wesenszüge an die Stelle eines naiven Dualismus-Begriffs.

5. Der Dualismus wird als historische Motivation für die Fortbildung genutzt.

6. Einblicke in Ergebnisse aus aktueller Forschung werden gewünscht, sollten aber nicht im Zentrum der Fortbildung stehen.

Schlüsselexperimente haben besonders im Bereich der Quantenphysik die Funktion, Vertrauen in die Theoriebildung zu schaffen. Quantenobjekte verhalten sich grundlegend anders als klassische Objekte, daher sollte auch aus Sicht der Lehrenden ein Zugang zu den Quantenphänomenen mit Experimenten geschaffen werden. Die Ergebnisse einer Sortieraufgabe innerhalb der Delphi-Studie unterstreichen dies zusätzlich: Befragte sortieren Themen, die eine besondere Motivation zur Teilnahme an der Lehrerfortbildung darstellen $(N=53)$. Fünf der angegebenen Themen konnten von den Teilnehmenden ausgewählt und absteigend nach Interesse sortiert werden. Die mehrheitlich nicht gewählten Themen stehen gleichberechtigt auf dem sechsten Rang. Ein Medianrang wurde berechnet. In Tab. 2 sind die Ergebnisse dargestellt. Über 50\% der Lehrenden haben Realexperimente auf den ersten Rang gewählt.

Die Ergebnisse stützen ebenfalls die obengenannte zweite Leitlinie: Über die Hälfte der Teilnehmenden wählen die Wesenszüge der Quantenphysik auf den dritten oder einen noch höheren Rang. Bereits zuvor wurde die Bedeutung der Wesenszüge als fachdidaktisches Konzept hervorgehoben. Dies ist auch darin zu begründen, dass mit dem Konzept zahlreiche Materialien angeboten werden, die direkt in den 
Tab. 2 Sortierung zu motivierenden Themen für eine Fortbildung zum Thema Quantenphysik

\begin{tabular}{ll}
\hline Thema & Median \\
\hline Realexperimente in der Quantenphysik & 1 \\
Die Wesenszüge der Quantenphysik & 3 \\
Simulationen in der Quantenphysik & 4 \\
Überblick über die verschiedenen Deutungsmöglichkei- & 4 \\
$\begin{array}{l}\text { ten der Quantenphysik } \\
\text { Zugänge zur Unschärferelation }\end{array}$ & 5 \\
$\begin{array}{l}\text { Der Einsatz des Computers im Themengebiet Quanten- } \\
\text { physik }\end{array}$ & 6 \\
$\begin{array}{l}\text { Unterrichtliche Einbindung der Schrödingergleichung } \\
\text { Umgang mit dem Paradigmenwechsel }\end{array}$ & 6 \\
Zeigerformalismus & 6 \\
\hline
\end{tabular}

Unterricht einfließen können. Die Nähe zur Unterrichtspraxis ist für die Teilnehmenden ein wichtiger Faktor.

Nachfolgende Items aus der dritten Delphi-Runde unterstreichen dies und stützen somit zusätzlich die dritte Leitlinie (siehe Abb. 10).

Eine Minimalanforderung an die Fortbildung ist offenbar, dass die Teilnehmenden mit mindestens einer konkreten Unterrichtsidee die Fortbildung verlassen (NIdee, $\mathrm{Zu}$ stimmung $96 \%$ ). Unmittelbar einsetzbare Unterrichtsmaterialien stellen ebenfalls einen starken Attraktor dar (UMat, Zustimmung $82 \%$ ).

Es zeigte sich weiterhin, dass die Diskussion um den Dualismus-Begriff in der Schulpraxis nicht eindeutig ab- geschlossen ist. Eine Vermeidung des Dualismus-Begriffs im Rahmen der Fortbildung scheint kein gangbarer Weg zu sein. Es sollte der Sinn der Vermeidung im Unterricht herausgearbeitet und die Relation zu den Wesenszügen der Quantenphysik verdeutlicht werden. Hierzu bietet es sich an, Teilchen- und Wellencharakter als Motivation zu nutzen, also die Unteilbarkeit an optischen Grenzflächen und die Interferenzfähigkeit herauszustellen.

Auch zeigt sich ein hohes eigenes Interesse der Teilnehmenden an fachlichen Themen aus der Forschung. Themen aktueller Forschung sollten in die Fortbildung einfließen. Die Teilnehmenden haben ein großes Interesse an den Themen Quantencomputer, Quantenkryptographie, Verschränkung (Abb. 11, Zustimmung jeweils über 60\%). Der Fokus sollte jedoch auf den Themen liegen, die in den Unterricht einfließen können, die im Kerncurriculum gefordert werden und sich somit an der Schulpraxis orientieren.

Insgesamt muss das Ziel der Fortbildung weit über eine Vermittlung der Konzepte hinausgehen. Konkrete Unterrichtsgänge, die eng mit den Schlüsselexperimenten der Quantenphysik verknüpft sind, sollen im Vordergrund stehen. Diese werden im Rahmen der Fortbildung als Realexperimente angeboten, auch wenn eine Durchführung in der Schule derzeit noch nicht möglich ist. Darüber hinaus wurden der Austausch und die Verknüpfung mit den Kolleginnen und Kollegen, sowie der Austausch von Unterrichtsmaterialien als wichtige Elemente der Fortbildung hervorgehoben.

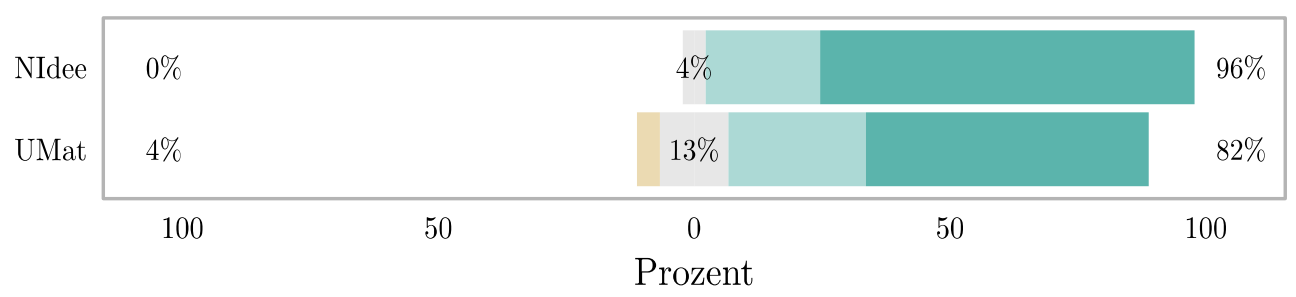

Stimme nicht zu Stimme eher nicht zu Neutral Stimme eher zu Stimme zu

Abb. 10 Ein hohes $\mathrm{Maß}$ an praxisrelevanten Inhalten wird eingefordert (NIdee mindestens eine neue Unterrichtsidee als Anforderung an die Fortbildung, UMat es muss Material für mindestens eine Unterrichtsstunde bereitgestellt werden; die genaue Itemformulierung kann dem Anhang entnommen werden)

Abb. 11 Teilnehmende haben Interesse an Themen aktueller Forschung (QComp Quantencomputer, aktFoQ aktuelle Forschung im Bereich der Quantenphysik, QKrypt Quantenkryptographie, Verschr Verschränkung; die genaue Itemformulierung kann dem Anhang entnommen werden)

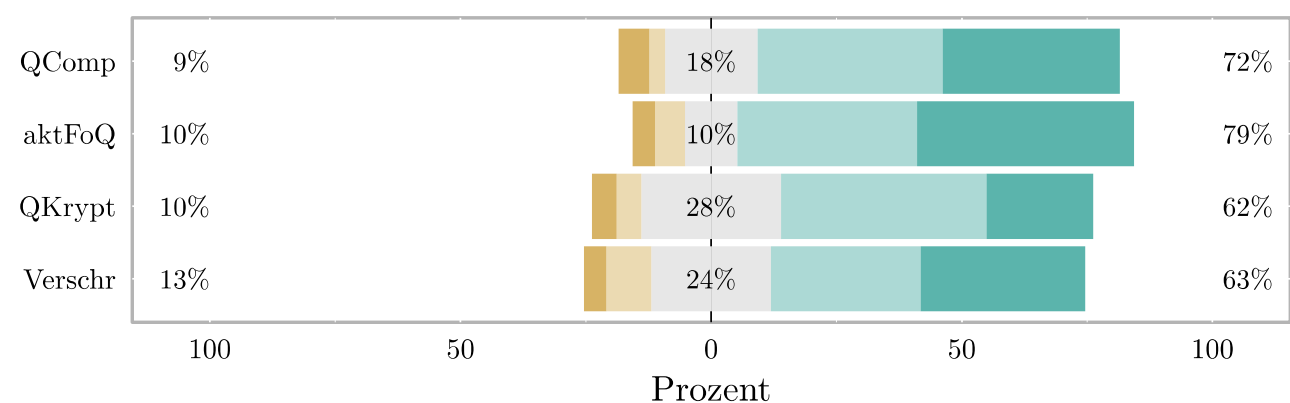

$\begin{array}{lllll}\text { Stimme nicht } \mathrm{zu} & \text { Stimme eher nicht zu Neutral } & \text { Stimme eher zu } & \text { Stimme zu }\end{array}$ 


\section{Diskussion der Ergebnisse}

Es wurde eine empirische Basis über die Sicht von Lehrenden auf Schulebene auf fachdidaktische Konzepte im Themenbereich der Quantenphysik geschaffen. Zusammen mit der Analyse der fachdidaktischen Konzepte konnten Leitlinien für eine Lehrerfortbildung konstruiert werden. Eine systematisch an den Bedarf von Lehrerinnen und Lehrern angepasste Fortbildung, die Lehrende im Bereich der Quantenphysik unterstützt, war das Resultat.

Die Delphi-Methode eignete sich dabei zur Erhebung der Ansichten der Lehrenden im besonderen Maße, da der iterative Charakter die wechselseitige Interaktion des didaktischen Triplets im ERTE-Modell unterstützt.

Folgende Limitationen liegen vor:

- Rolle des Monitoring-Teams

- Subjektivität in der Wahrnehmung des eigenen Unterrichts

- Bildungspolitischer Rahmen und Verallgemeinerung

- Stichprobenumfang und Selektionseffekte

- Zeitraum der Studie

\section{Rolle des Monitoring-Teams}

Das Monitoring-Team bestand aus den drei Autoren dieses Beitrags. Das Team konnte sich nicht nur auf eine überwachende Tätigkeit beschränken, sondern musste auch steuernd in den Verlauf der Delphi-Studie eingreifen. Zwei Eingriffe haben stattgefunden: Fokussierung auf den Bereich Quantenobjekte und Ausklammern des Themengebiets Zeigerformalismus nach der zweiten Runde.

Mit der Fokussierung auf den Bereich Quantenobjekte entfällt der Bereich Atomphysik. Insbesondere wird das Thema „Anschauungen zu Atommodellen“ ausgeklammert. Die Entscheidung beruht darauf, dass im Bereich Quantenobjekte ein Anknüpfen an aktuellen Themen der Physik möglich ist und dieser Bereich einen besonderen Beitrag zu „Nature of Science“ leisten kann.

Der Themenstrang Zeigerformalismus wurde in der dritten Delphi-Runde nicht weiterverfolgt. Die zahlreichen Beiträge der ersten und zweiten Delphi-Runde zeigten, dass der Erfolg dieser Repräsentation von zahlreichen Rahmenbedingungen abhängt: Der parallel erfahrene Mathematikunterricht, der Einsatz der Zeigerdarstellung im Themenbereich „Schwingungen und Wellen“ sowie die Einstellung zu möglichen alternativen Darstellungsformen spielen eine wesentliche Rolle für den Erfolg des Einsatzes im Unterricht. Der Einsatz und Erfolg der Zeigerdarstellung müssen daher zum Gegenstand gesonderter Betrachtungen werden.

\section{Subjektive Wahrnehmung des eigenen Unterrichts}

Die vorgestellte Studie erhebt die subjektiven Ansichten zu Konzeptionen und konkretem Quantenphysik-Unterricht. Aussagen über den Unterricht, wie er in den Klassenräumen stattfindet, können hieraus nicht abgeleitet werden. Die Ergebnisse aus den Bereichen, welche die Wahrnehmung besonderer Herausforderungen bei den Lernenden erhebt, geben somit keine Evidenz für die tatsächlichen Schwierigkeiten, mit denen Lernende beim Lernen der Quantenphysik konfrontiert ist. Um diese zu erheben, müsste der Unterricht selbst und die Vorstellungen und Lernprozesse von Lernenden untersucht werden. Dennoch ist es wichtig, Erkenntnisse über die subjektive Sicht der Lehrenden zu gewinnen. Schließlich sind sie die potenziellen Teilnehmenden der Fortbildung.

\section{Bildungspolitischer Rahmen und Verallgemeinerung}

Die Curricula der Länder in Deutschland unterscheiden sich stark. Die vorgestellte Studie wurde in Niedersachsen durchgeführt. Das niedersächsische Curriculum (Chrost et al. 2009) zeichnet sich durch eine besondere Schwerpunktsetzung aus: Der Anteil des Themenbereichs Quantenobjekte im Oberstufencurriculum ist sehr ausgeprägt. Auch die Forderung nach einer quantitativen Betrachtung der Phänomene, bei der die Verwendung der Zeigerdarstellung nahegelegt wird, ist ein Alleinstellungsmerkmal im bundesweiten Vergleich. Diese besondere bildungspolitische Rahmung färbt die Ansichten der Lehrenden, sodass eine Übertragung der Ergebnisse auf andere Bundesländer im Einzelnen geprüft werden muss.

\section{Stichprobenumfang und Selektionseffekte}

Insgesamt wurden 254 Schulen in Niedersachsen angeschrieben. Dies entspricht allen öffentlichen Schulen mit einer gymnasialen Oberstufe. Geht man davon aus, dass etwa vier Lehrende regelmäßig den Oberstufenunterricht übernehmen, so repräsentieren die 75 Teilnehmenden der letzten Delphi-Stufe etwa 5-10\% der potentiellen Fortbildungsteilnehmenden. Hierdurch entsteht natürlich ein Selektionseffekt: Es ist davon auszugehen, dass eher die Lehrenden an der Delphi-Studie teilnehmen, die ein besonderes Interesse an didaktischer Forschung oder am Themenfeld Quantenphysik haben. Das Expertenpanel repräsentiert somit nicht die Grundgesamtheit der niedersächsischen Oberstufenlehrer. Die Teilnahme an Fortbildungen in Niedersachsen erfolgt jedoch freiwillig, sodass die Selektion den Pool der potentiellen Teilnehmenden gut repräsentieren dürfte. 


\section{Zeitraum der Studie}

Die beschriebene Studie wurde bereits vor fünf Jahren durchgeführt. In der Zwischenzeit hat sich das Niedersächsische Kerncurriculum geändert, sodass sich im Speziellen einzelne Aussagen auf das alte Kerncurriculum beziehen. Das neue Kerncurriculum (Frenzel et al. 2017) hat jedoch eine noch intensivere Schwerpunktbildung im Bereich der Quantenphysik erfahren. Somit ist das bearbeitete Themengebiet weiterhin von großem Interesse und die hier vorgestellten Ergebnisse vollständig übertragbar.

\section{Ausblick}

Mit den erarbeiteten Leitlinien kann eine initiale Fortbildungsstufe für einen Design-Based-Research-Ansatz erstellt werden. Ein möglicher Experimentepfad, der den Anforderungen gerecht wird, ist in der Literatur beschrieben (vgl. Scholz et al. 2018, 2016; Weber 2018). Ziel ist es, die Fortbildung weiterzuentwickeln, sodass die Transferwirkung optimiert wird. Im Vordergrund muss allerdings eine konkret operative Phase stehen, welche die Ergebnisse der Studie in der Praxis absichert. Dabei sollte nicht eine standardisierte Evaluation, sondern die dezidierte Betrachtung der Transferwirkung in den Unterricht im Vordergrund stehen.

Danksagung Wir danken den Gutachtern für die wertvollen Hinweise und Korrekturen.

Funding Open Access funding enabled and organized by Projekt DEAL.

\section{Anhang}

\section{Zu Abb. 4}

Arbeitsauftrag: (Aufzählung der Wesenszüge nach Küblbeck und Müller) Bitte bewerten Sie die nachstehenden Paraphrasen zu den Wesenszügen.

\begin{tabular}{lll}
\hline Abkürzung & Volltext & $N$ \\
\hline DiskuWesz & $\begin{array}{l}\text { Die Wesenszüge eröffnen in meinem Un- } \\
\text { terricht rege Diskussionen zur Quanten- }\end{array}$ & $58(\mathrm{eA})$ \\
& physik & \\
RoFWesz & $\begin{array}{l}\text { Die Wesenszüge ziehen sich wie ein „roter } \\
\\
\text { Faden“ durch meinen Unterricht }\end{array}$ & $57(\mathrm{eA})$ \\
& $48(\mathrm{gA})$ \\
\hline
\end{tabular}

\section{Zu Abb. 6}

Arbeitsauftrag: Bitte bewerten Sie folgende Aussagen zum Dualismus.

\begin{tabular}{lll}
\hline Abkürzung & Volltext & $N$ \\
\hline ModBi & $\begin{array}{l}\text { Der Dualismus ist ein gutes Beispiel, um } \\
\text { die Rolle von Modellbildung in der Physik }\end{array}$ & $56(\mathrm{eA})$ \\
& $47(\mathrm{gA})$ \\
zu thematisieren & \\
DiskSpFi & $\begin{array}{l}\text { Die Diskussion um den Dualismus-Begriff } \\
\text { ist „,Spitzfindig“. An der Uni wird der Be- } \\
\text { griff viel „natürlicher“ verwendet als dies }\end{array}$ & $39(\mathrm{eA})$ \\
& $33(\mathrm{gA})$ \\
& $\begin{array}{l}\text { in manchen didaktischen Diskussionen der } \\
\text { Fall ist }\end{array}$ & \\
ObjErfW & $\begin{array}{l}\text { Problematisch ist, dass der Begriff Dualis- } \\
\text { mus die Vorstellung von einem „Objekt“ }\end{array}$ & $46(\mathrm{eA})$ \\
& $\begin{array}{l}\text { aus der Erfahrungswelt der Lernenden } \\
\text { nahelegt; was Quantenobjekte aber nicht } \\
\text { sind }\end{array}$ & \\
&
\end{tabular}

\section{Zu Abb. 7}

Aufschlüsselung der absoluten Häufigkeiten.

\begin{tabular}{ll}
\hline Name & Häufigkeit \\
\hline Wesenszüge & 19 \\
1D Potentiale & 12 \\
Zeigerformalismus & 10 \\
Interpretationen QP & 6 \\
Gesamte QP & 5 \\
Formalismus & 5 \\
Welle-Teilchen-Dualismus & 4 \\
Kernmodelle & 4 \\
Schrödingergleichung & 4 \\
Welcher Weg Experiment & 3 \\
Franck-Hertz-Versuch & 3 \\
Unschärferelation & 2 \\
Alphazerfall & 1 \\
De-Broglie-Wellenlänge & 1 \\
Elektronenbeugung & 1 \\
Impuls & 1 \\
\hline
\end{tabular}

\section{Zu Abb. 8}

Arbeitsauftrag: (Aufzählung der Wesenszüge nach Küblbeck und Müller) Bitte bewerten Sie die nachstehenden Paraphrasen zu den Wesenszügen.

\begin{tabular}{lll}
\hline Abkürzung & Volltext & $N$ \\
\hline RExpWesz & $\begin{array}{l}\text { Der schulrelevante Teil der Wesenszüge } \\
\text { sollte durch Realexperimente unterstützt } \\
\text { werden }\end{array}$ & $60(\mathrm{eA})$ \\
SimuWesz & $\begin{array}{l}\text { Simulationsprogramme veranschaulichen } \\
\text { die einzelnen Wesenszüge gut }\end{array}$ & $61(\mathrm{eA})$ \\
RelImAbitur Der Zeitaufwand für die SuS bei der Er- & $52(\mathrm{gA})$ \\
& $54(\mathrm{eA})$ \\
& $\begin{array}{l}\text { arbeitung der Wesenszüge steht in keinem } \\
\text { Verhältnis zu deren bisheriger Relevanz im }\end{array}$ & $46(\mathrm{gA})$ \\
& Abitur & \\
\hline
\end{tabular}




\section{Zu Abb. 9}

Aufschlüsselung der absoluten Häufigkeiten.

\begin{tabular}{ll}
\hline Name & $N$ \\
\hline Experimente & 15 \\
Didaktisierung der QP & 9 \\
Quantenphysik allgemein & 7 \\
Zeigerformalismus & 5 \\
Weniger Formalismus & 4 \\
1D Potentiale & 4 \\
Schrödingergleichung & 3 \\
Verschränkung & 3 \\
Nichtlokalität & 3 \\
Komplementarität & 3 \\
Verbindung Experiment Theorie & 2 \\
Mit QP Ausbildung zufrieden & 2 \\
Heisenbergsche Matrizenmechanik & 1 \\
Historie & 1 \\
Atommodelle & 1 \\
Messprozess & 1 \\
\hline
\end{tabular}

\section{Zu Abb. 10}

Arbeitsauftrag: Bitte bewerten Sie die nachstehenden Aussagen zu den Anforderungen an das Bildungsangebot.

\begin{tabular}{lll}
\hline Abkürzung & Volltext & $N$ \\
\hline NIdee & $\begin{array}{l}\text { Ich muss mit mindestens einer konkret } \\
\text { umsetzbaren, neuen Unterrichtsidee nach }\end{array}$ & 67 \\
& $\begin{array}{l}\text { Hause gehen } \\
\text { UMat }\end{array}$ & $\begin{array}{l}\text { Das Bildungsangebot muss Unterrichtsma- } \\
\text { terialien für mindestens eine umsetzbare }\end{array}$ \\
& 67 \\
Unterrichtsstunde bereitstellen & \\
\hline
\end{tabular}

\section{Zu Abb. 11}

Arbeitsauftrag: Sollten Anwendungen zur Quantenphysik in dem Bildungsangebot behandelt werden, soll dies adressatengerecht erfolgen. Bitte bewerten Sie die folgenden Aussagen vor diesem Hintergrund.

In einem Bildungsangebot möchte ich etwas über ... erfahren.

\begin{tabular}{lll}
\hline Abkürzung & Volltext & $N$ \\
\hline QComp & Quantencomputer & 65 \\
aktFoQ & Aktuelle Forschung in der Quantenphysik & 67 \\
QKrypto & Quantenkryptographie & 61 \\
Verschr & Verschränkung & 67 \\
\hline
\end{tabular}

Open Access Dieser Artikel wird unter der Creative Commons Namensnennung 4.0 International Lizenz veröffentlicht, welche die Nutzung, Vervielfältigung, Bearbeitung, Verbreitung und Wiedergabe in jeglichem Medium und Format erlaubt, sofern Sie den/die ursprünglichen Autor(en) und die Quelle ordnungsgemäß nennen, einen Link zur Creative Commons Lizenz beifügen und angeben, ob Änderungen vorgenommen wurden.

Die in diesem Artikel enthaltenen Bilder und sonstiges Drittmaterial unterliegen ebenfalls der genannten Creative Commons Lizenz, sofern sich aus der Abbildungslegende nichts anderes ergibt. Sofern das betreffende Material nicht unter der genannten Creative Commons Lizenz steht und die betreffende Handlung nicht nach gesetzlichen Vorschriften erlaubt ist, ist für die oben aufgeführten Weiterverwendungen des Materials die Einwilligung des jeweiligen Rechteinhabers einzuholen.

Weitere Details zur Lizenz entnehmen Sie bitte der Lizenzinformation auf http://creativecommons.org/licenses/by/4.0/deed.de.

\section{Literatur}

Ammon, U. (2009). Delphi-Befragung. In S. Kühl, P. Strodtholz \& A. Taffertshofer (Hrsg.), Handbuch Methoden der Organisationsforschung (S. 458-476). Wiesbaden: VS.

Bader, F. (2000). Eine Quantenwelt - ohne Dualismus (Naturwissenschaftlicher Unterricht heute: Physik, Sekundarbereich II). Hannover: Schroedel.

Brachner, A., \& Fichtner, R. (1977). Quantenmechanik für Lehrer und Studenten. Hannover: Schroedel.

Bronner, P. (2010). Quantenoptische Experimente als Grundlage eines Curriculums zur Quantenphysik des Photons. Studien zum Physik- und Chemielernen, Bd. 103. Berlin: Logos Verlag.

Bronner, P., Strunz, A., Silberhorn, C., \& Meyn, J.-P. (2009). Interactive screen experiments with single photons. European Journal of Physics, 30(2), 345.

Bryer, J., \& Speerschneider, K. (2016). Likert-R Package

Burkard, U. (2009). Quantenphysik in der Schule: Bestandsaufnahme, Perspektiven und Weiterentwicklungsmöglichkeiten durch die Implementierung eines Medienservers. Studien zum Physik- und Chemielernen, Bd. 90. Berlin: Logos Verlag.

Chrost, G., Gehrmann, K., Hampe, U., Heider, M., Mannigel, N., Marx, G., et al. (2009). Kerncurriculum gymnasiale Oberstufe Physik Niedersachsen. Hannover: Niedersächsisches Kultusministerium.

Darling-Hammond, L., Wei, R.C., Andree, A., Richardson, N., \& Orphanos, S. (2009). Professional learning in the learning profession: a status report on teacher development in the U.S. and abroad. Technical report. National staff development council. http://www. eric.ed.gov/ERICWebPortal/detail?accno=ED504168. Zugegriffen: 25. Apr. 2020.

Van Dijk, E.M., \& Kattmann, U. (2007). A research model for the study of science teachers' PCK and improving teacher education. Teaching and Teacher Education, 23(6), 885-897.

Erb, R., \& Schön, L. (1997). Ein Blick in den Spiegel - Einblick in die Optik. In Handlungs- und kommunikationsorientierter Unterricht in der Sek. II (S. 30-54).

Feynman, R.P. (2006). QED: die seltsame Theorie des Lichts und der Materie. München: Piper.

Feynman, R.P., Leighton, R.B., Sands, M.L., \& Gottlieb, M.A. (1963). The Feynman lectures on physics III. The Feynman Lectures on Physics. Addison-Wesley: Pearson.

Fischler, H. (1992). Quantenphysik in der Schule. Kiel: IPN.

Fischler, H., \& Lichtfeldt, M. (1992). Learning quantum mechanics. Research in physics learning: theoretical issues and empirical studies (S. 240-258). Kiel: IPN. 
Frenzel, M., Gössing, J., Hampe, U., Haubrock, D., Krökel, P., Müller, T., et al. (2017). Kerncurriculum gymnasiale Oberstufe Physik Niedersachsen. Hannover: Niedersächsisches Kultusministerium.

Gläser, J., \& Laudel, G. (2010). Experteninterviews und qualitative Inhaltsanalyse. Wiesbaden: VS.

Häder, M. (2009). Delphi-Befragungen: Ein Arbeitsbuch. Wiesbaden: VS.

Häussler, P., Frey, K., Hoffmann, L., Rost, J., \& Spada, H. (1980). Physikalische Bildung: eine curriculare Delphi-Studie: Teil I: Verfahren und Ergebnisse. IPN-Arbeitsberichte. Kiel: IPN.

Kattmann, U., Duit, R., Gropengießer, H., \& Komorek, M. (1997). Das Modell der Didaktischen Rekonstruktion - Ein Rahmen für naturwissenschaftsdidaktische Forschung und Entwicklung. Zeitschrift für Didaktik der Naturwissenschaften, 3(3), 3-18.

Krüger, D., Parchmann, I., \& Schecker, H. (2014). Methoden in der naturwissenschaftsdidaktischen Forschung. Berlin: Springer.

Küblbeck, J., \& Müller, R. (2003). Die Wesenszüge der Quantenphysik: Modelle, Bilder, Experimente. Köln: Aulis-Verlag Deubner.

Kultusministerkonferenz (2004). Einheitliche Prüfungsanforderungen in der Abiturprüfung Physik. https://www.kmk.org/fileadmin/ veroeffentlichungen_beschluesse/1989/1989_12_01-EPA-Physik. pdf. Zugegriffen: 24. Mai 2020.

Kultusministerkonferenz (2020a). Bildungspläne/Lehrpläne der Länder im Internet. https://www.kmk.org/dokumentation-statistik/ rechtsvorschriften-lehrplaene/uebersicht-lehrplaene.html. Zugegriffen: 10. Juli 2020.

Kultusministerkonferenz (2020b). Bildungsstandards im Fach Physik für die Allgemeine Hochschulreife. https://www.kmk.org/ fileadmin/Dateien/veroeffentlichungen_beschluesse/2020/2020_ 06_18-BildungsstandardsAHR_Physik.pdf. Zugegriffen: 10. Juli 2020.

Lakatos, I. (1974). Falsifikation und die Methodologie wissenschaftlicher Forschungsprogramme. In Kritik und Erkenntnisfortschritt (S. 89-189). Berlin: Springer.

Landis, J.R., \& Koch, G. G. (1977). The Measurement of Observer Agreement for Categorical Data. Biometrics, 33(1), 159.

Laumann, D., Wichtrup, P., \& Friege, G. (2019). Zwei Schlüssel zur Physik - Reale Experimente und digitale Medien als Schlüssel zu physikalischen Inhalten. Unterricht Physik, 171/172, 4-10.

Leuchs, G., Bayer, M., Bloch, I., Boche, H., Bruß, D., Budker, D., et al. (2017). Grundlagenpapier QUTEGA: QUANTENTECHNOLOGIE - Grundlage und Anwendung. https://www. quantentechnologien.de/fileadmin/public/Redaktion/Dokumente/ PDF/Publikationen/Qutega-QT-Grundlagen-und-Anwendungen01-2017-C1.pdf. Zugegriffen: 24. Mai 2020.

Lipowsky, F., \& Rzejak, D. (2012). Lehrerinnen und Lehrer als Lerner - Wann gelingt der Rollentausch? Merkmale und Wirkungen wirksamer Lehrerfortbildungen. Reform der Lehrerbildung in Deutschland, Österreich und der Schweiz, 1, 235-253.

Müller, R. (2005). Qualitative Quantenphysik: Eine Handreichung für die Sekundarstufe I im Rahmen von piko. Physik im Kontext. Kiel: IPN.

Müller, R., \& Wiesner, H. (2000). Das Münchener Unterrichtskonzept zur Quantenmechanik. Physik in der Schule, 38, 126.

Müller, R., Wiesner, H., \& Hoff, H. (2000). Nutzung des Internets zur Lehrerfortbildung in Quantenmechanik. Der mathematischnaturwissenschaftliche Unterricht, 54(1), 23-26.
Nawrath, D. (2010). Kontextorientierung-Rekonstruktion einer fachdidaktischen Konzeption für den Physikunterricht. Oldenburg: BIS.

Niedderer, H. (1992). Atomphysik mit anschaulichen Quantenmodellen. In H. Fischler (Hrsg.), Quantenphysik in der Schule (S. 88-113). Kiel: IPN.

Pospiech, G., \& Schöne, M. (2012). Quantenphysik in Schule und Hochschule (S. 1-10).

Reinmann, G. (2005). Innovation ohne Forschung? Ein Plädoyer für den Design-Based Research-Ansatz in der Lehr-Lernforschung; Innovation without Research? Arguments for Design-Based Research in Educational Research. Unterrichtswissenschaft, 33(1), $52-69$.

Reisch, C., \& Franz, T. (2016). Quantenkryptographie. PdN PHYSIK in der Schule, 1, 11-17.

Robbins, N., \& Heiberger, R. (2011). Plotting Likert and Other Rating Scales. Proceedings of the 2011 Joint Statistical Meeting.

Rode, M. (2011). Biphotonen - ein modellierender Zugang zur Verschränkung. Der mathematische und naturwissenschaftliche Unterricht, 64(3), 149-152.

Rode, M. (2013). Quantenphysik in der Schule am Halbspiegel lernen. PdN PHYSIK in der Schule, 1(62), 15-23.

Rode, M. (2017). Argumente und falsche Freunde im Unterricht über Quantenphysik. In G. Friege \& R. Scholz (Hrsg.), Argumentieren in der Quantenphysik (S. 6-15). Braunschweig: Schroedel.

Rode, M., \& Barth, M. (2017). Quantenphysik. Unterricht Physik, 162.

Schäffer, B., \& Loos, P. (2001). Das Gruppendiskussionsverfahren. Wiesbaden: VS.

Schneider, J., \& Meyn, J.-P. (2016). Modellexperimente zur Quantenkryptographie. PdN Physik, 1, 36-39.

Scholz, R., Friege, G., \& Weber, K.-A. (2016). Undergraduate experiments on statistical optics. European Journal of Physics, 37(5), 55302.

Scholz, R., Friege, G., \& Weber, K. A. (2018). Undergraduate quantum optics-Experimental steps to quantum physics. European Journal of Physics, 37(5), 55301.

Weber, K.-A. (2018). Quantenoptik in der Lehrerfortbildung: Ein Bedarfsgeprägtes Fortbildungskonzept Zum Quantenobjekt, Photon Mit Realexperimenten. Studien Zum Physik- Und Chemielernen, Bd. 269. Berlin: Logos Verlag.

Welzel, M., Haller, K., Bandiera, M., Hammelev, D., Koumaras, P., Niedderer, H., et al. (1998). Ziel, die Lehrende mit dem Experimentieren in der naturwissenschaftlichten Ausbildung verbinden. Zeitschrift für Didaktik der Naturwissenschaften, 4, 29-44.

Werner, J. (2000). Vom Licht zum Atom: ein Unterrichtskonzept zur Quantenphysik unter Nutzung des Zeigermodells. Studien Zum Physik- Und Chemielernen, Bd. 12. Berlin: Logos.

Willer, J. (2003). Didaktik des Physikunterrichts. Frankfurt am Main: Harri Deutsch.

Wolf, W., Göbel-Lehnert, U., \& Chroust, P. (1999). Fortbildung der Lehrerinnen und Lehrer: eine Bilanz ihrer Formen und Wirkungen anhand empirischer Untersuchungen. Die Deutsche Schule, 91, 451-467.

Zollman, D. A., Rebello, N.S., \& Hogg, K. (2002). Quantum mechanics for everyone: Hands-on activities integrated with technology. American Journal of Physics, 70(3), 252-259. 